\title{
Saccharin salts of active pharmaceutical ingredients, their crystal structures and increased water solubilities
}

Rahul Banerjee, ${ }^{\dagger}$ Prashant M. Bhatt, ${ }^{\dagger}$ Nittala V. Ravindra, ${ }^{\ddagger}$ and Gautam R. Desiraju ${ }^{* \dagger}$

Contribution from the School of Chemistry, University of Hyderabad, Hyderabad 500046, India (gautam_desiraju@yahoo.com) and Informatics Division, GVK Biosciences Private Limited, 6-3-1192,Begumpet, Hyderabad 500 016, India.

\footnotetext{
${ }^{\dagger}$ University of Hyderabad.

${ }^{\ddagger}$ GVK Biosciences Private Limited.
}

\section{Supporting Information}

(25 pages)

For each API saccharinate, the following details are provided.

(i) ORTEP plot (50\% probability for non-H atoms).

(ii) Rietveld refinement of experimental (red) and calculated (blue) X-ray powder patterns.

(iii) DSC trace of recrystallised sample (pure).

(iv) DSC trace of ground sample (generally impure).

(v) IR details of samples (before and after solubility determination)

For mirtazapine saccharinate, hot stage microscope photographs are additionally provided. 


\section{Haloperidol saccharinate:}

(i)

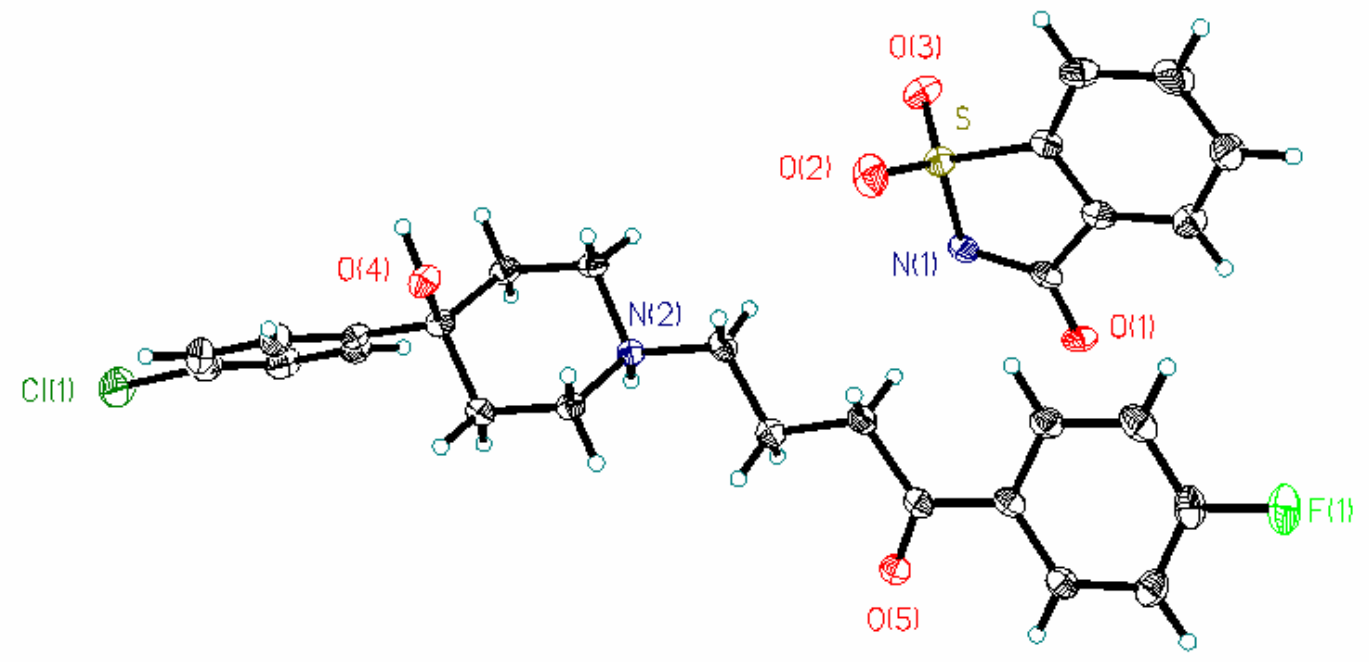

(ii)

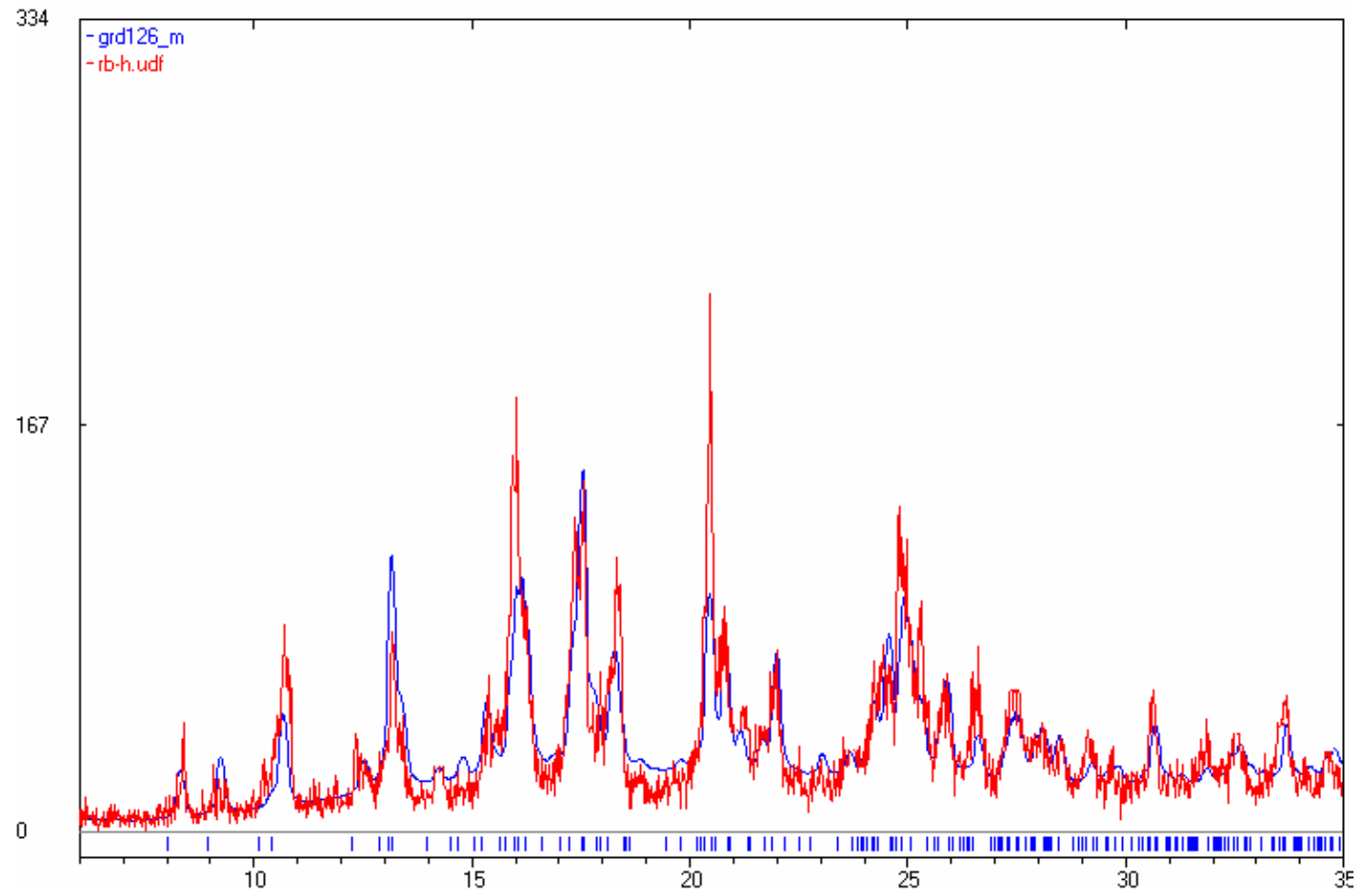


(iii)

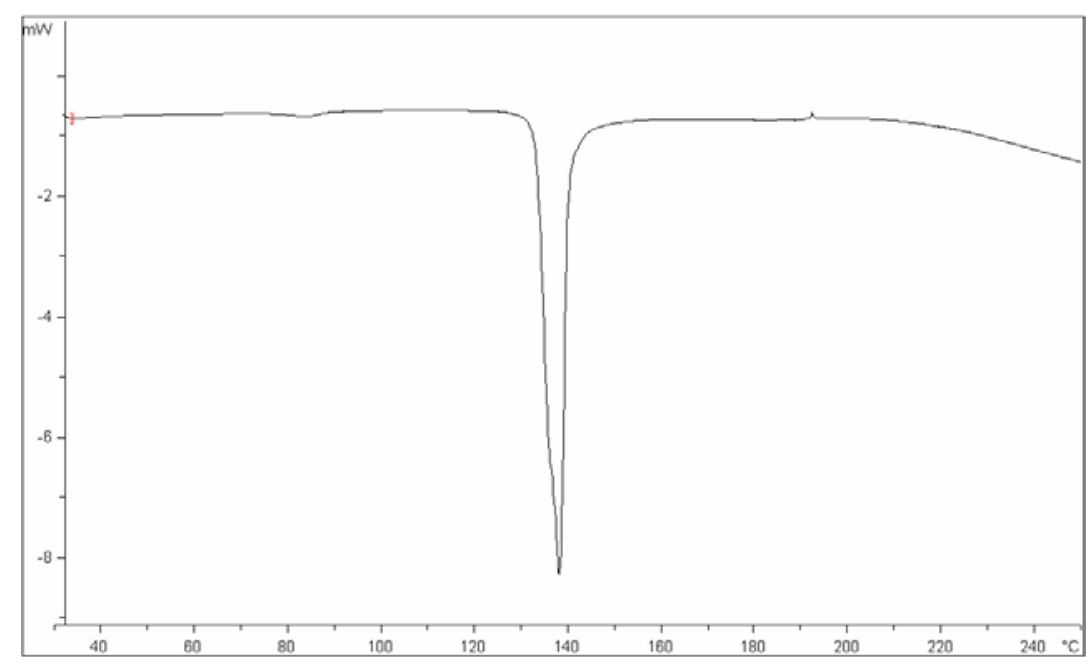

(iv)

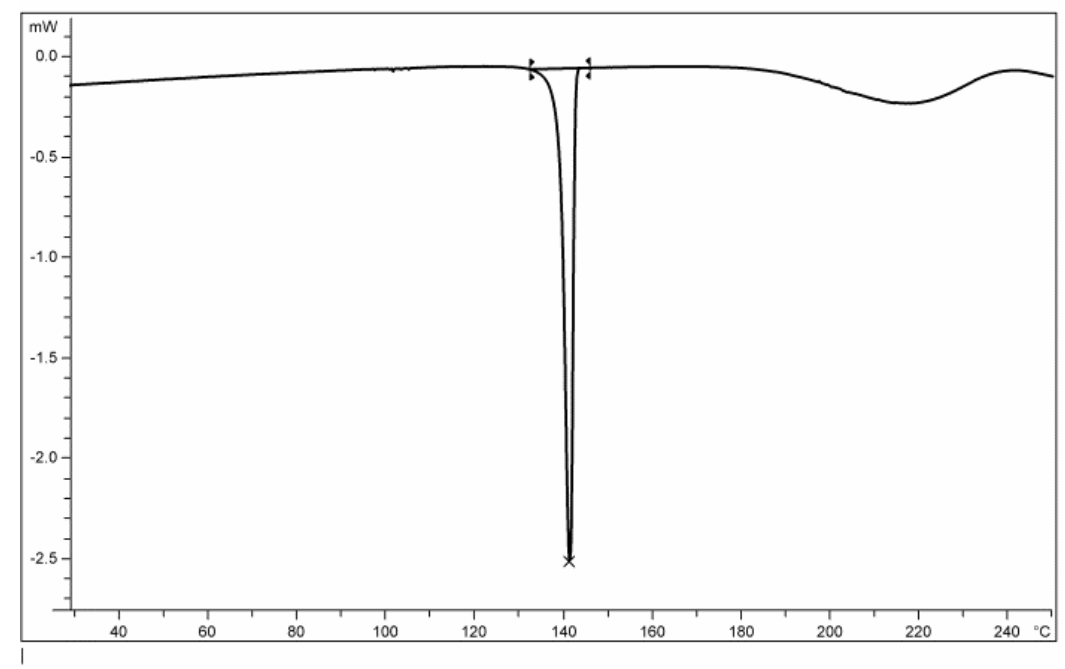

(v)

Saccharin $\left(\mathrm{cm}^{-1}\right)$ : 3396, 3094, 2972, 2690, 2010, 1975, 1865, 1720, 1593, 1460, 1336, $1255,1178,1138,1118,1008,972,898,794,758,702$.

Haloperidol $\left(\mathrm{cm}^{-1}\right)$ : 3130, 2955, 2922, 2822, 1682, 1599, 1410, 1361, 1219, 1155, 1045, 997, 960, 918, 829.

Haloperidol saccharinate (before solubility) $\left(\mathrm{cm}^{-1}\right)$ : 3393, 2982, 2939, 2897, 2723, 2613, 1684, 1628, 1581, 1479, 1410, 1263, 1147, 1043, 983, 949, 831, 752.

Haloperidol saccharinate (after solubility) $\left(\mathrm{cm}^{-1}\right)$ : 3391, 2984, 2725, 2604, 1684, 1628, 1581, 1479, 1410, 1263, 1147, 1043, 983, 949, 831, 752. 


\section{Pseudoephedrine saccharinate:}

(i)

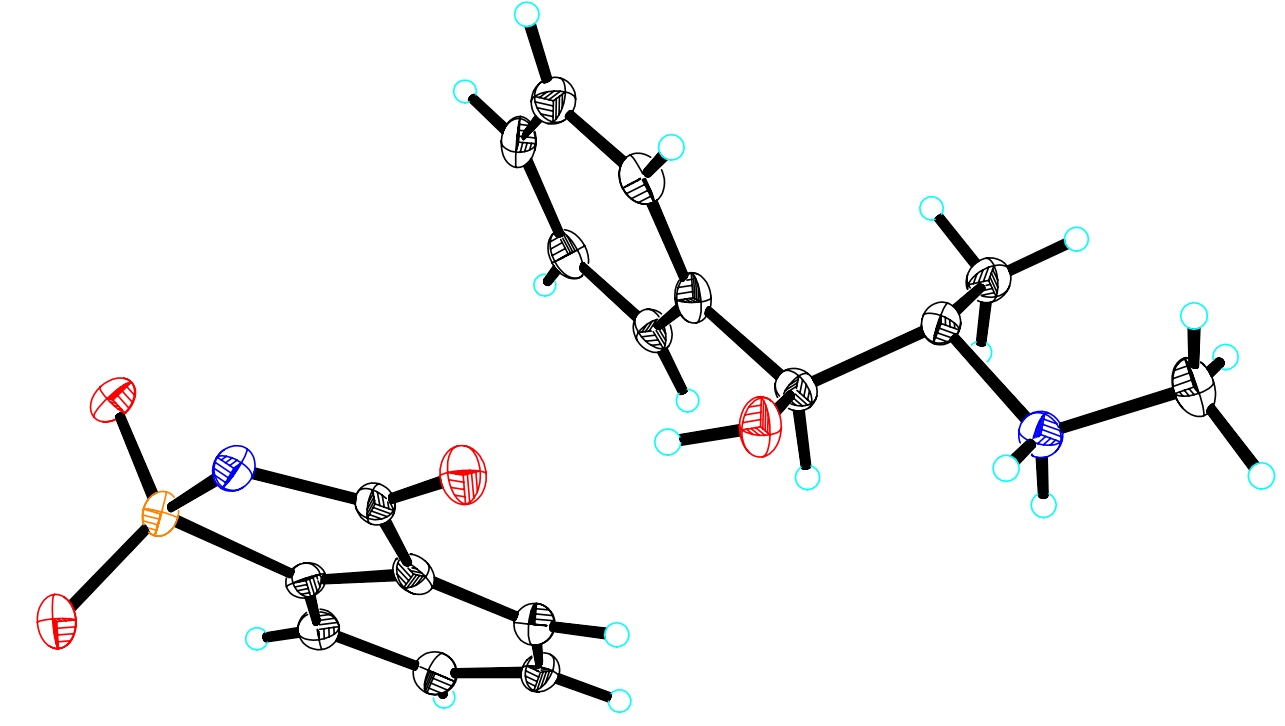

(ii)

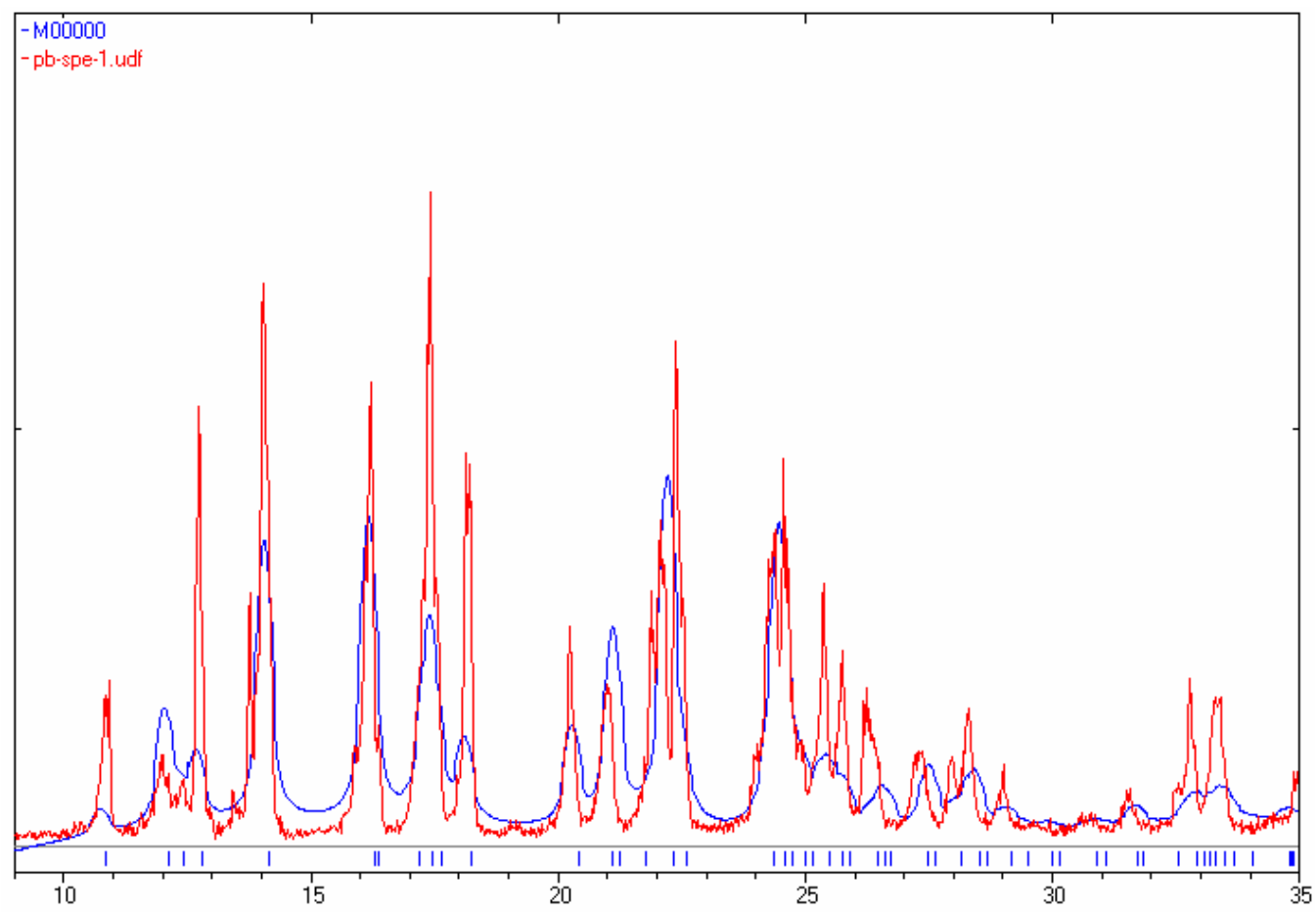


(iii)

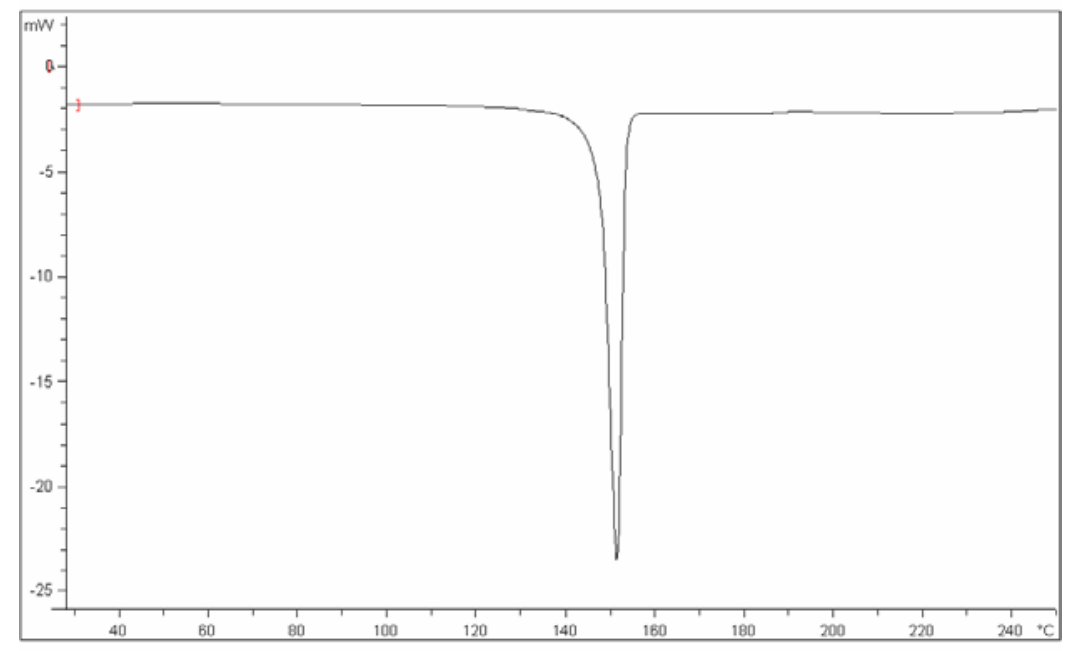

(iv)

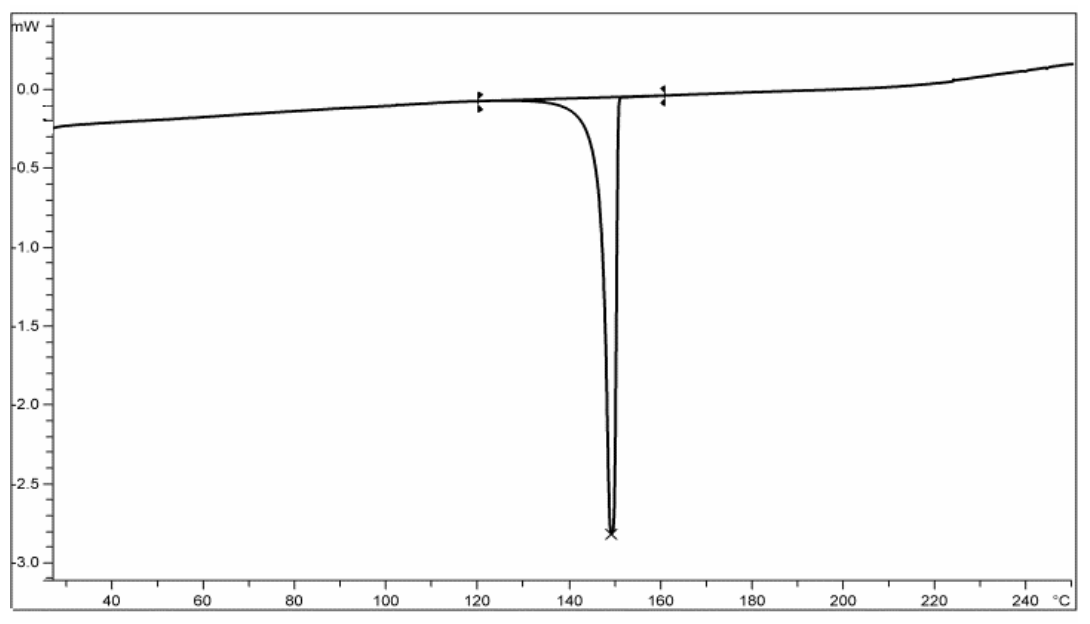

(v)

Saccharin $\left(\mathrm{cm}^{-1}\right)$ : 3396, 3094, 2972, 2690, 2010, 1975, 1865, 1720, 1593, 1460, 1336, 1255, 1178, 1138, 1118, 1008, 972, 898, 794, 758, 702.

Pseudoephedrine $\left(\mathrm{cm}^{-1}\right)$ : 3319, 3055, 2976, 2802, 1988, 1822, 1699, 1601, 1558, 1479, 1437, 1375, 1338, 1288, 1257, 1203, 1155, 1093, 1062, 1022, 947, 910, 852, 800, 761.

Pseudoephedrine saccharinate (before solubility) $\left(\mathrm{cm}^{-1}\right)$ : 3296, 3240, 3190, 2991, 2719, 2488, 2451, 1639, 1587, 1458, 1392, 1267, 1145, 1037, 951, 773.

Pseudoephedrine saccharinate (after solubility) $\left(\mathrm{cm}^{-1}\right)$ : 3290, 3238, 3190, 2993, 2721, 2488, 2447, 1639, 1587, 1458, 1331, 1267, 1145, 1037, 951, 773. 


\section{Lamivudine saccharinate:}

(i)

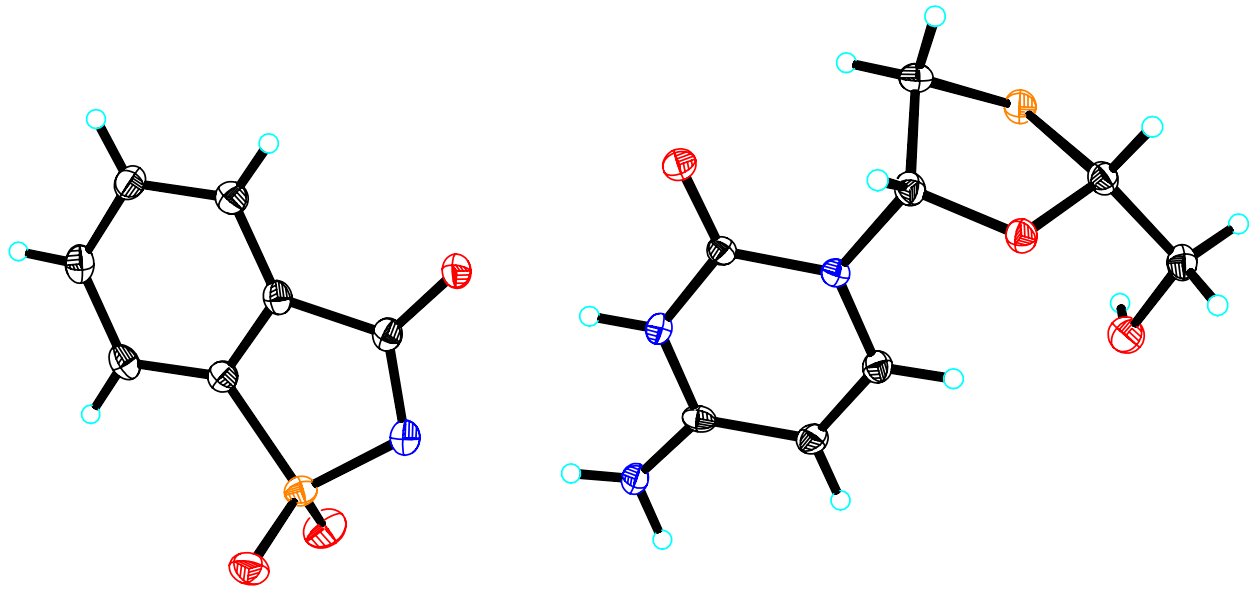

(ii)

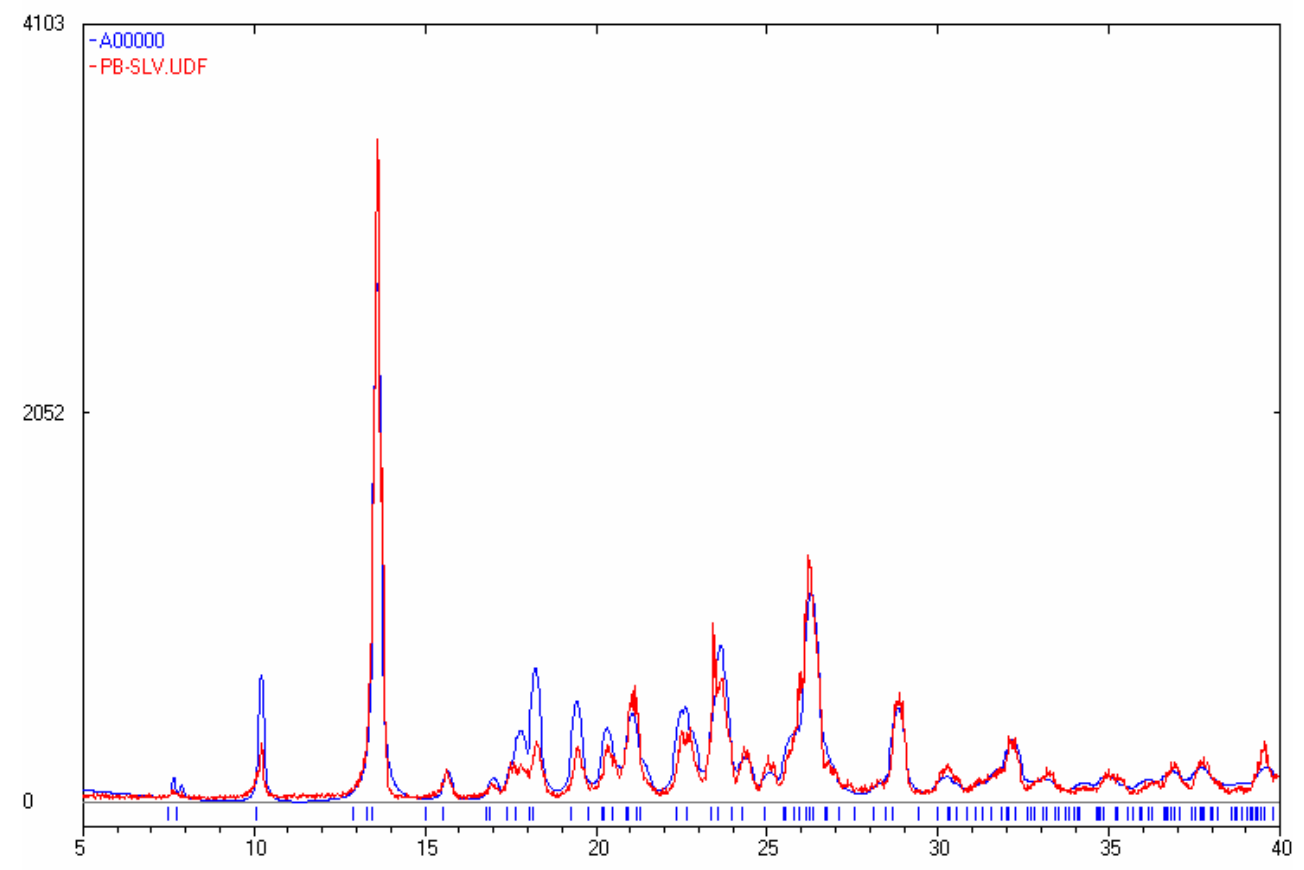


(iii)

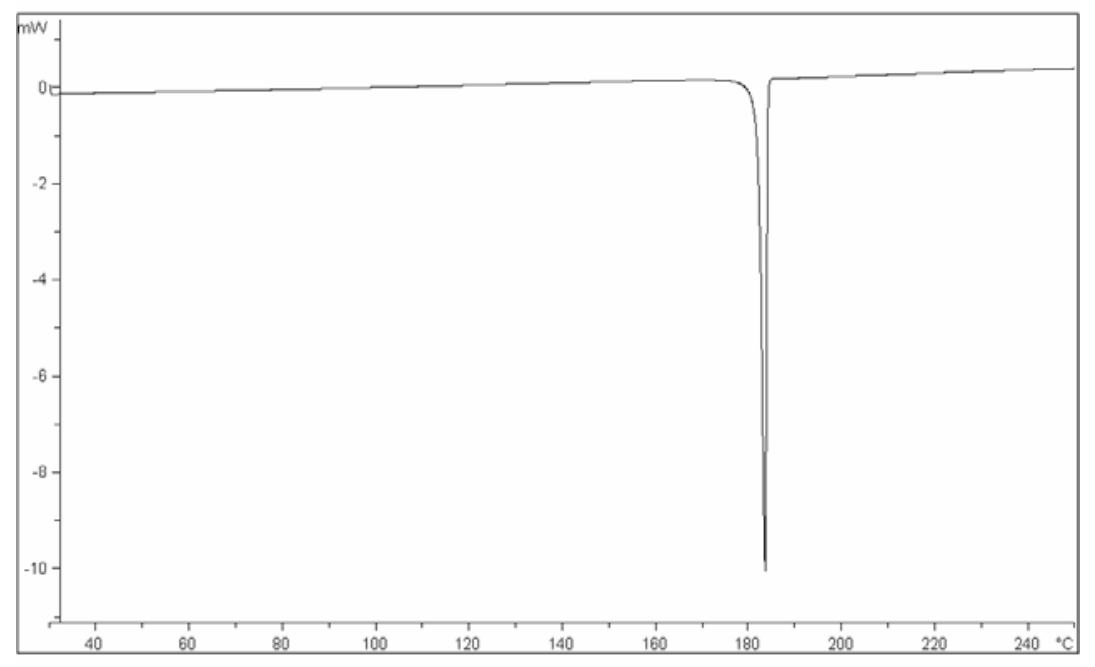

(iv)

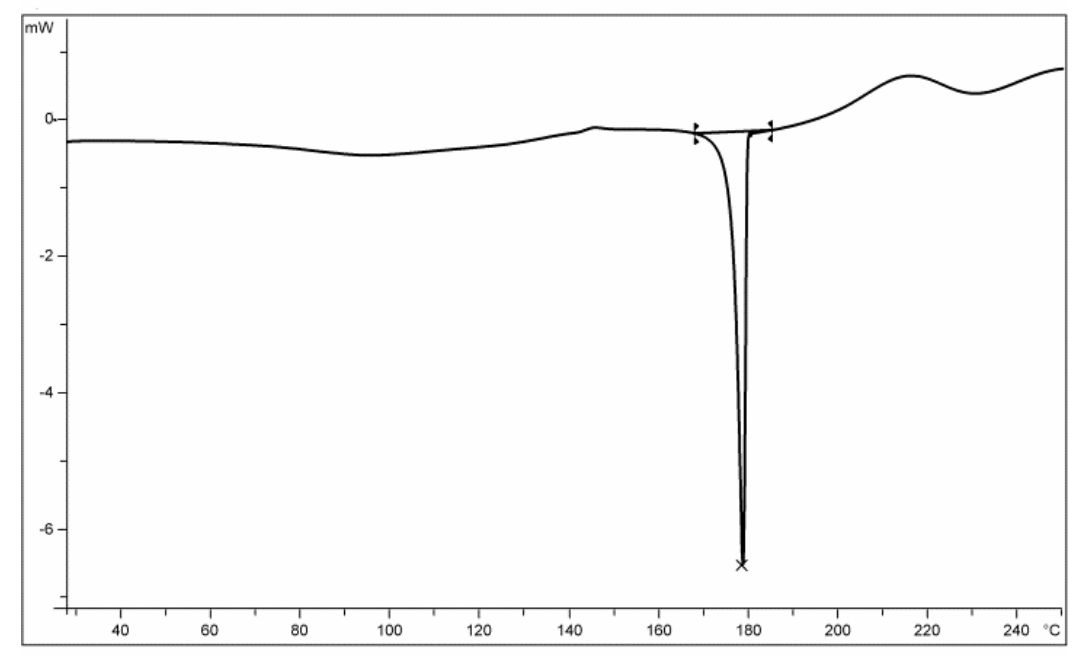

(v)

Saccharin $\left(\mathrm{cm}^{-1}\right)$ : 3396, 3094, 2972, 2690, 2010, 1975, 1865, 1720, 1593, 1460, 1336, 1255, 1178, 1138, 1118, 1008, 972, 898, 794, 758, 702.

Lamivudine $\left(\mathrm{cm}^{-1}\right)$ : 3331, 3204, 1651, 1635,1606, 1496, 1396, 1286, 1159, 1087, 1059, 1028, 916, 850.

Lamivudine saccharinate (before solubility) $\left(\mathrm{cm}^{-1}\right)$ : 3456, 3288, 3026, 1921, 1697, 1641, 1545, 1456, 1402, 1358, 1273, 1143, 1059, 1010, 943, 814, 748.

Lamivudine saccharinate (after solubility) $\left(\mathrm{cm}^{-1}\right)$ : 3458, 3287, 3024, 1934, 1697, 1641, 1545, 1458, 1402, 1358, 1273, 1145, 1059, 1016, 943, 814, 748. 
Powder pattern of lamivudine saccharinate a) before and b) after solubility

a)
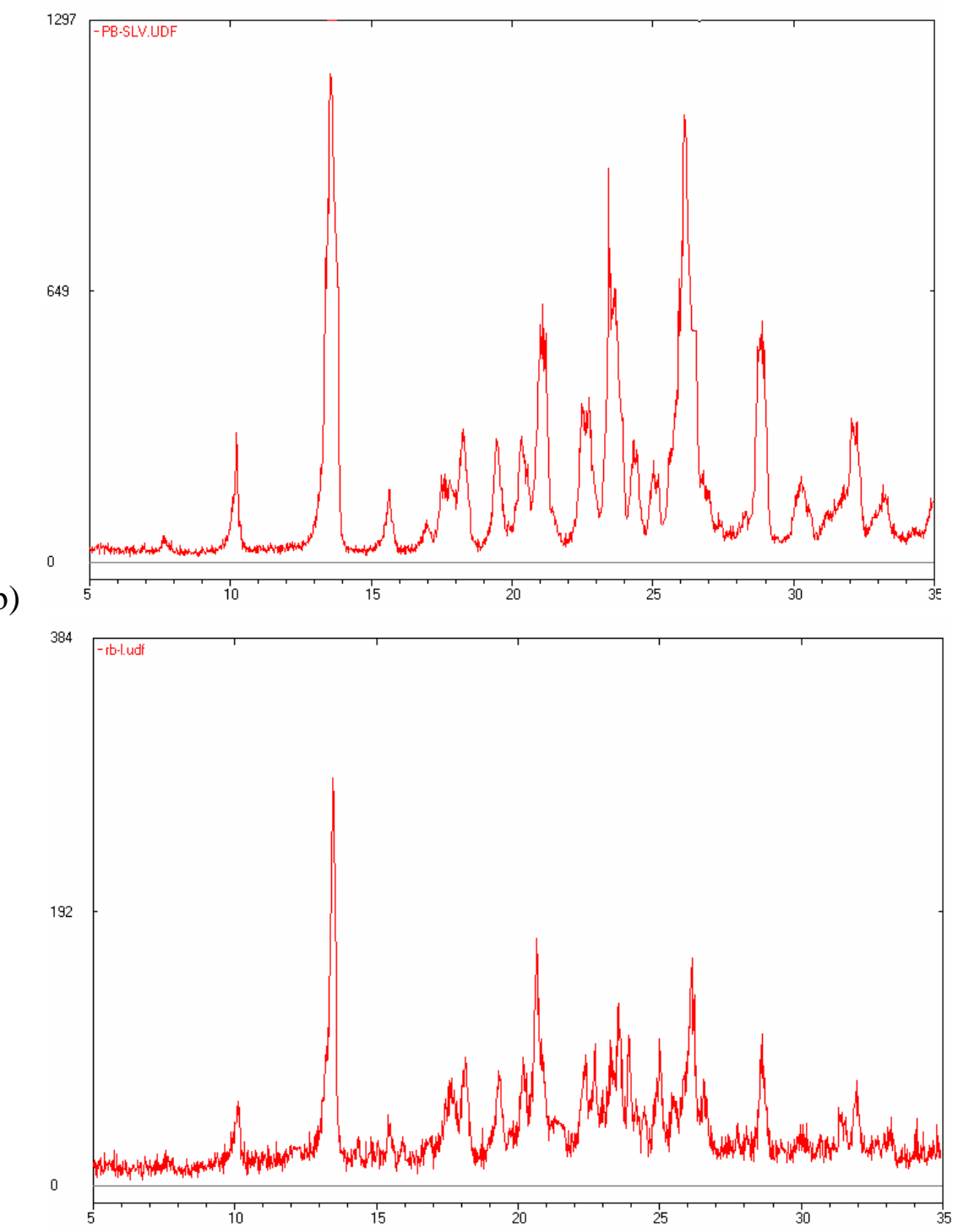


\section{Risperidone saccharinate:}

(i)

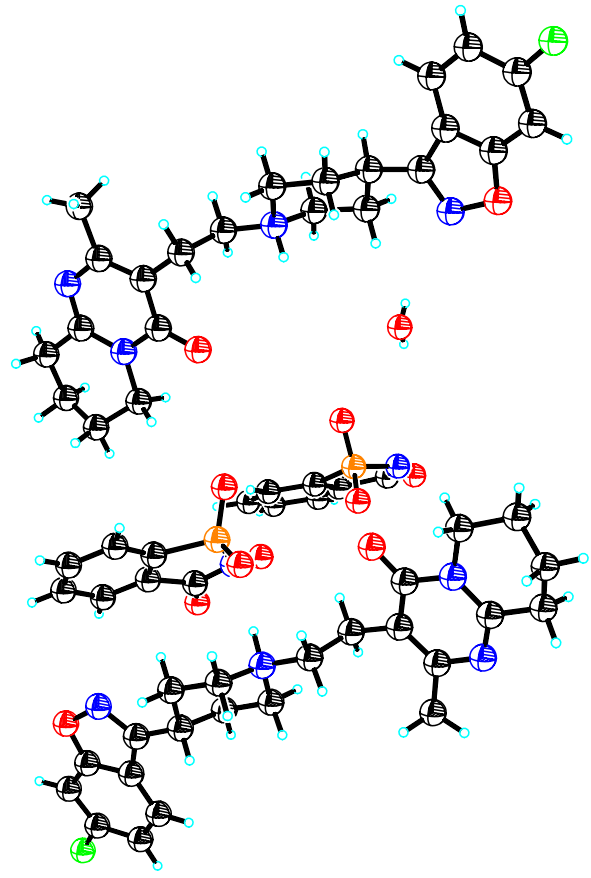

(ii)

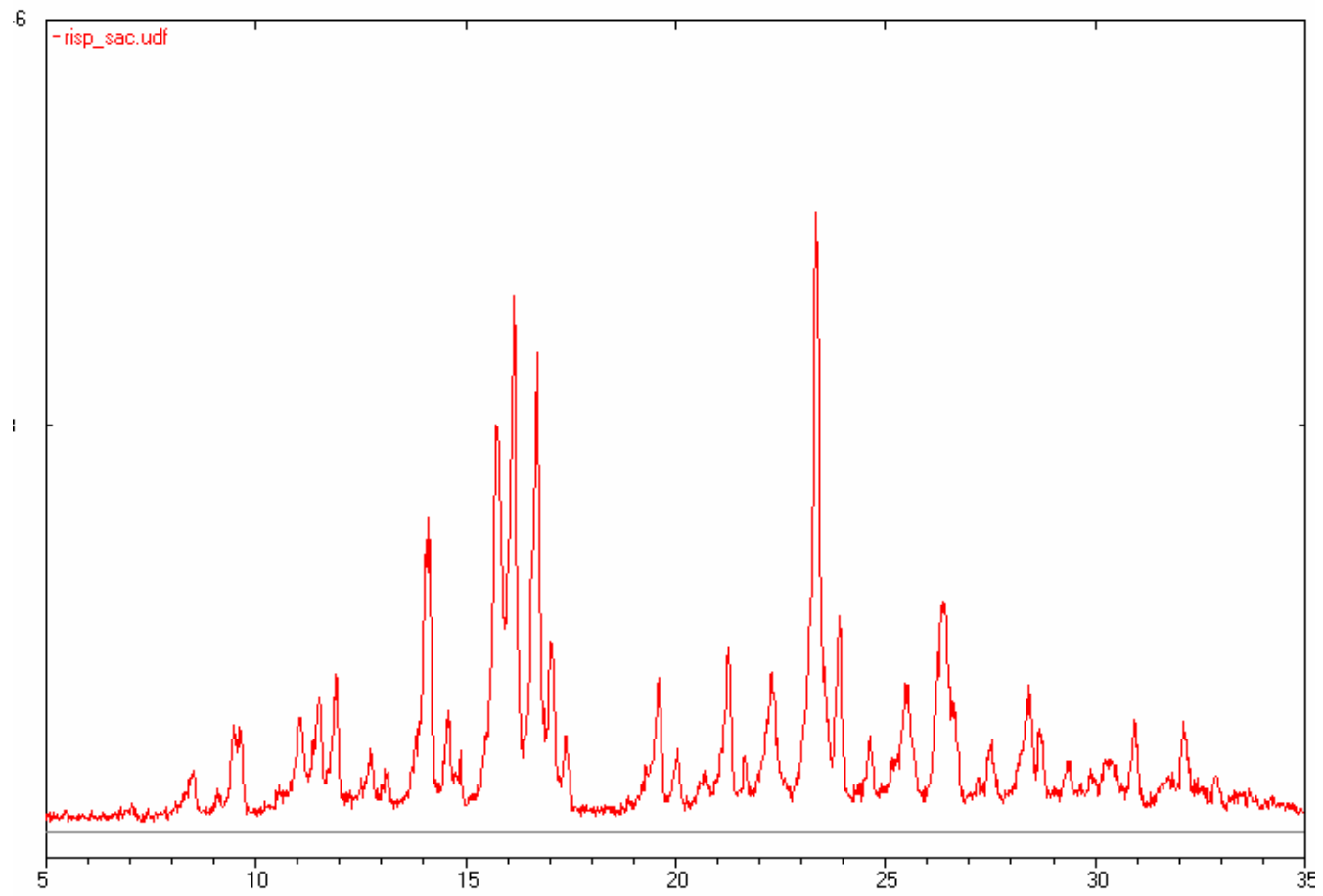

Rietveld profile fit is not possible as we so far are unable to get the crystal structure of anhydrous risperidone saccharinate. 
(iii)

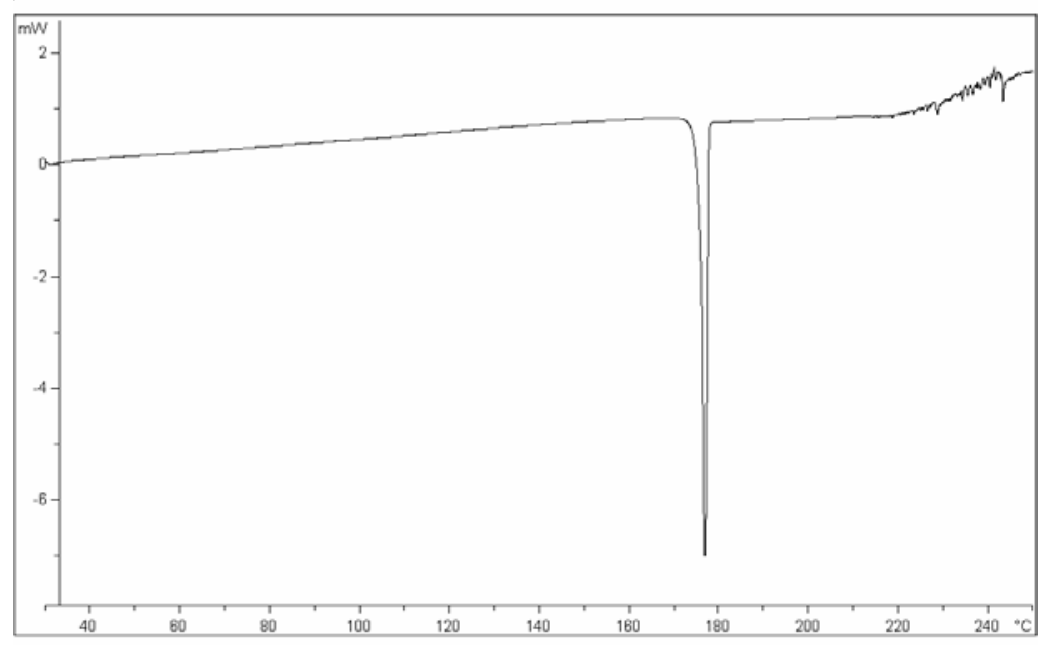

(iv)

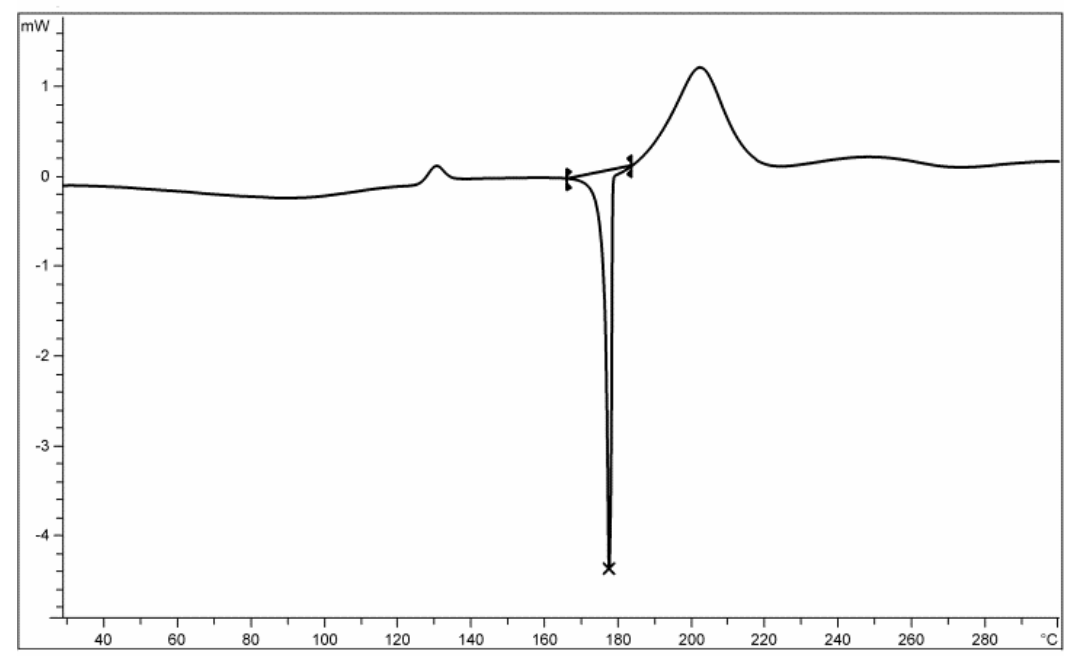

(v)

Saccharin $\left(\mathrm{cm}^{-1}\right)$ : 3396, 3094, 2972, 2690, 2010, 1975, 1865, 1720, 1593, 1460, 1336, $1255,1178,1138,1118,1008,972,898,794,758,702$.

Risperidone $\left(\mathrm{cm}^{-1}\right)$ : 3063, 2943, 2758, 1649, 1606, 1535, 1413, 1350, 1309, 1269, 1192, 1130, 1024, 993, 958, 864, 815.

Risperidone saccharinate (before solubility) $\left(\mathrm{cm}^{-1}\right)$ : 3547, 3420,3067, 2970, 2544, 1645, 1585, 1529, 1450, 1327, 1275, 1151, 1114, 1053, 949, 823, 750.

Risperidone saccharinate (after solubility) $\left(\mathrm{cm}^{-1}\right)$ : 3539, 3420, 3076, 2968, 2557, 1645, 1585, 1523, 1450, 1327, 1275, 1151, 1114, 1053, 949, 823, 750. 


\section{Venlafaxine saccharinate:}

(i)

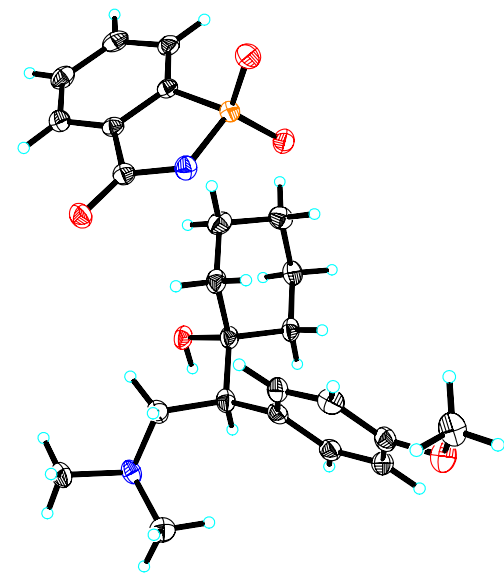

(ii)

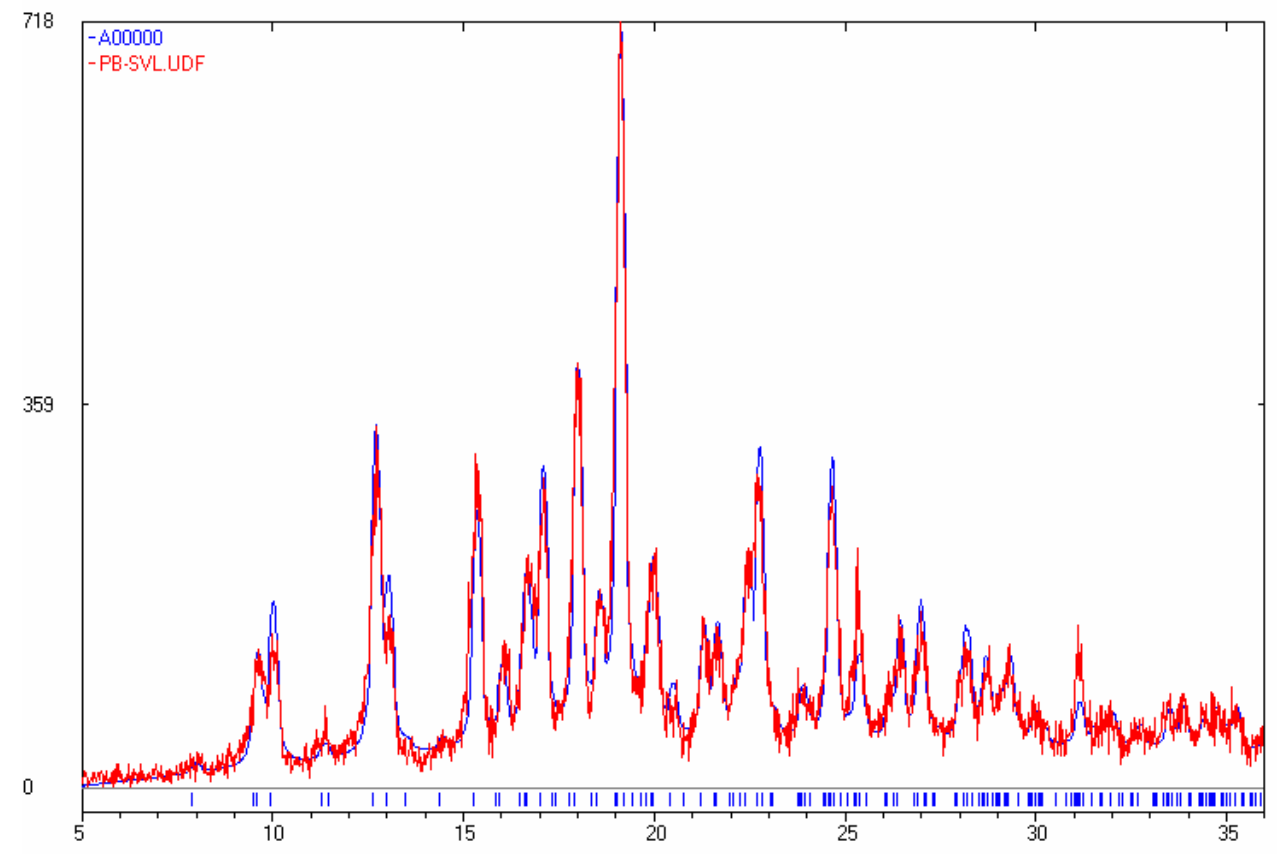


(iii)

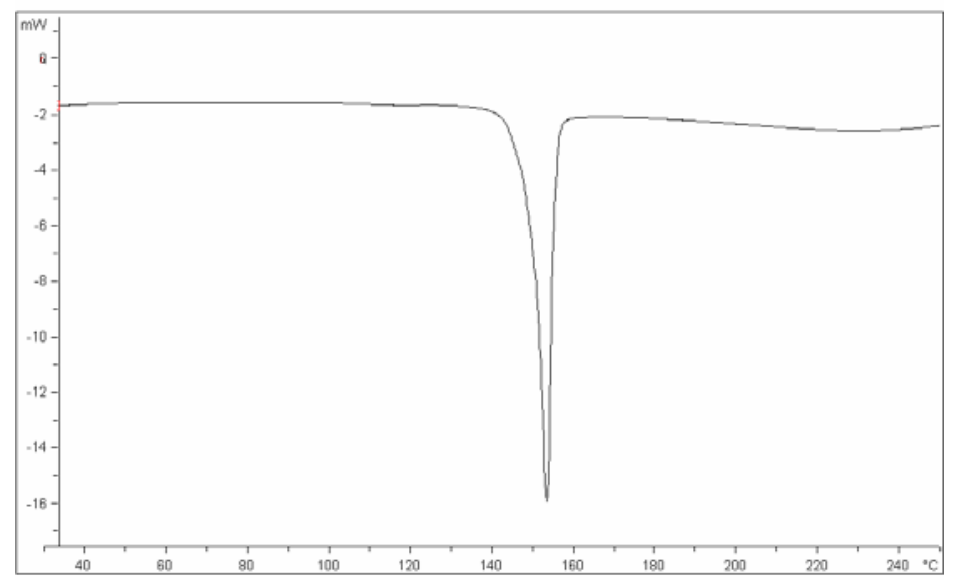

(iv)

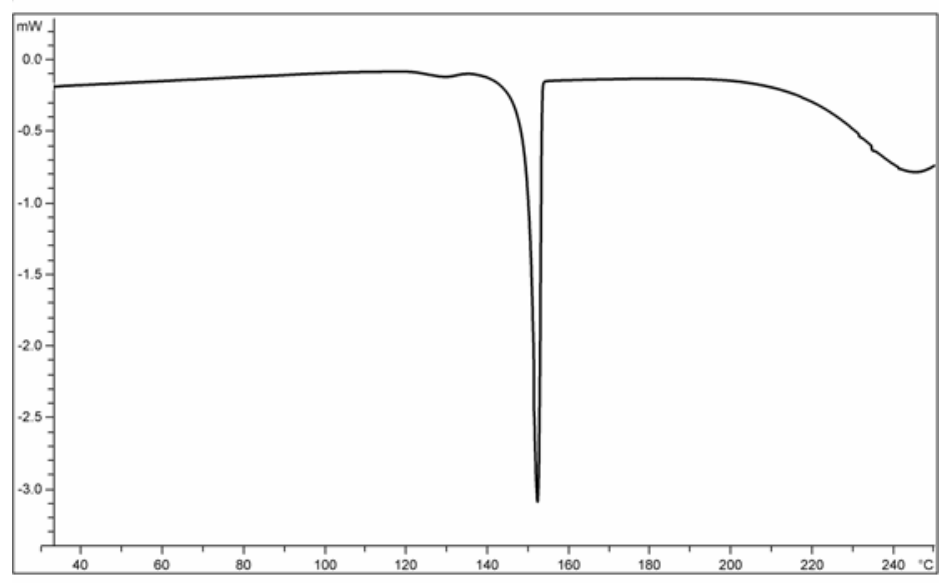

(v)

Saccharin $\left(\mathrm{cm}^{-1}\right)$ : 3396, 3094, 2972, 2690, 2010, 1975, 1865, 1720, 1593, 1460, 1336, 1255, 1178, 1138, 1118, 1008, 972, 898, 794, 758, 702.

Venlafaxine ( $\left.\mathrm{cm}^{-1}\right)$ : 3157, 3038, 2945, 2829, 2779, 2069, 1853, 1608, 1579, 1514, 1464, 1440, 1334, 1280, 1240, 1178, 1145, 1103, 1074, 1039, 1010, 968, 925, 904.

Venlafaxine saccharinate (before solubility) $\left(\mathrm{cm}^{-1}\right)$ : 3258, 2945, 2854, 2592, 2517, 2422, 1917, 1635,1583, 1516, 1456, 1332, 1282, 1248, 1153, 1030, 947, 842, 758.

Venlafaxine saccharinate (after solubility) $\left(\mathrm{cm}^{-1}\right)$ : 3256, 2945, 2854, 2521, 2422, 1915, $1633,1583,1516,1456,1332,1282,1248,1157,1030,947,842,760$ 
Powder pattern of venlafaxine saccharinate a) before b) after solubility

a)

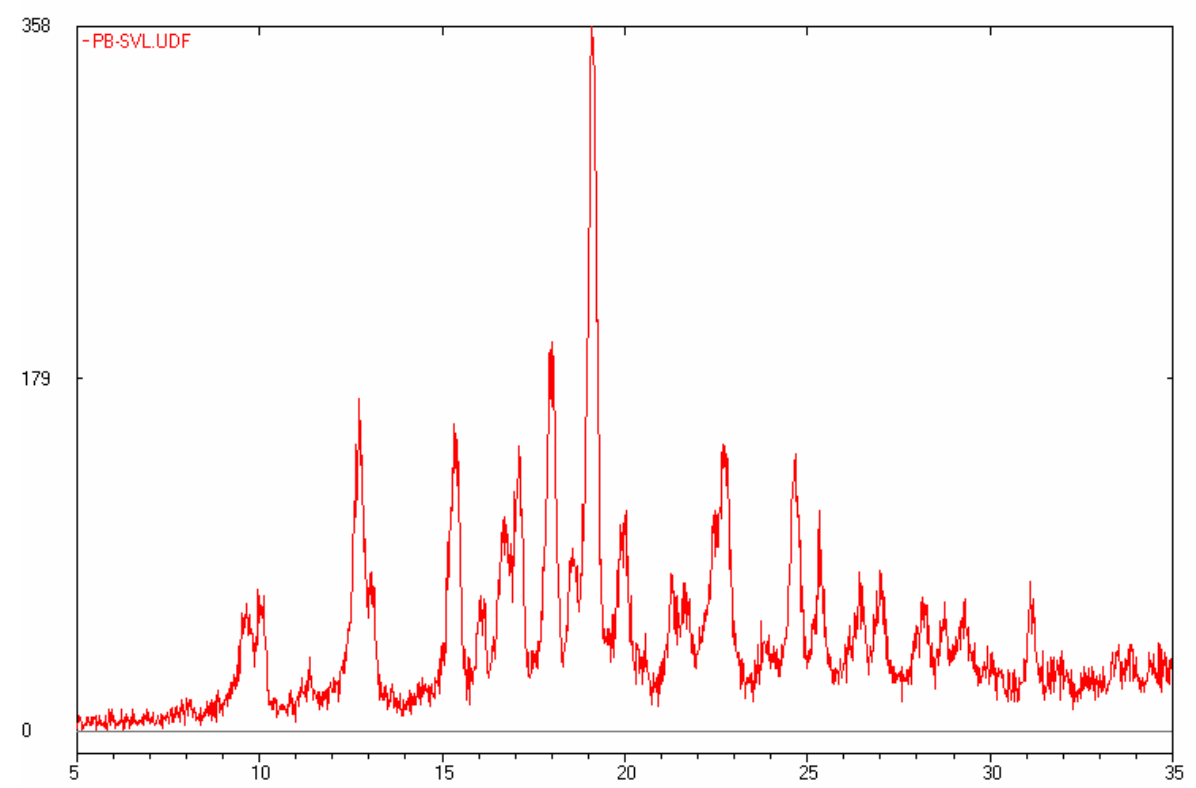

b)

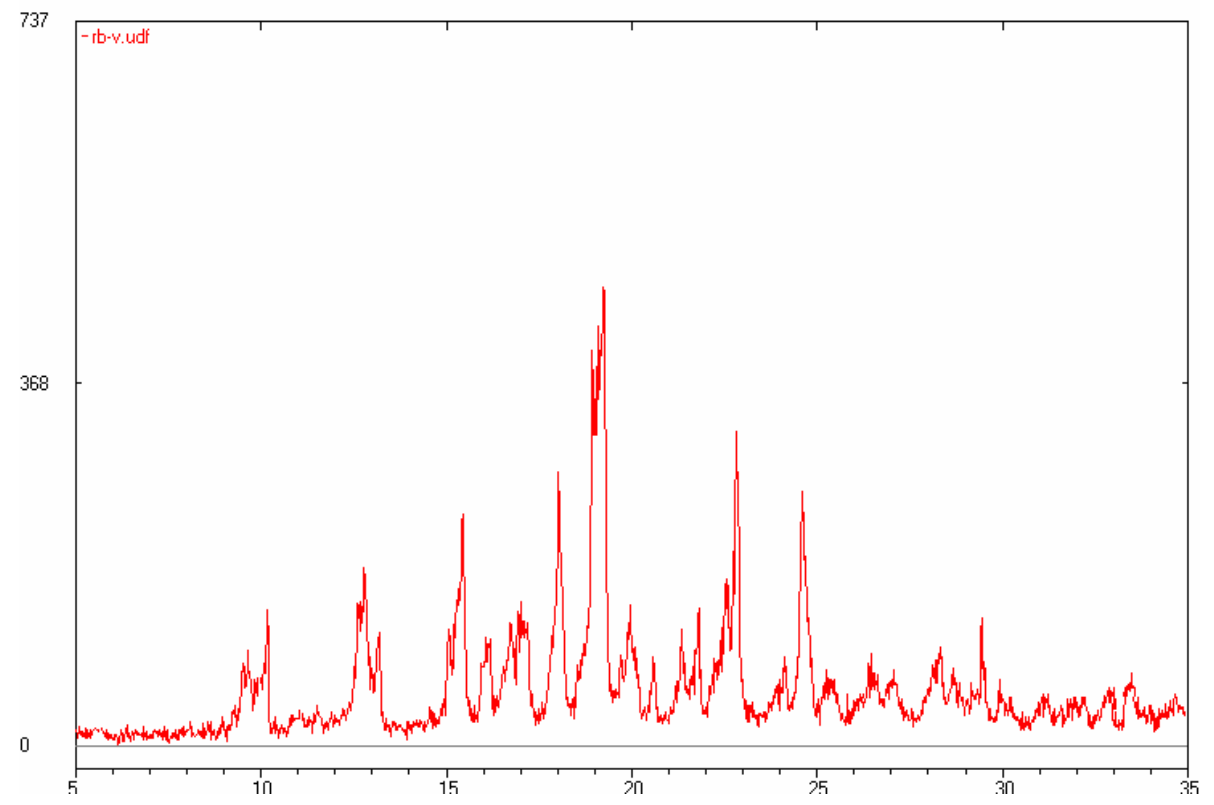




\section{Zolpidem saccharinate:}

(i)

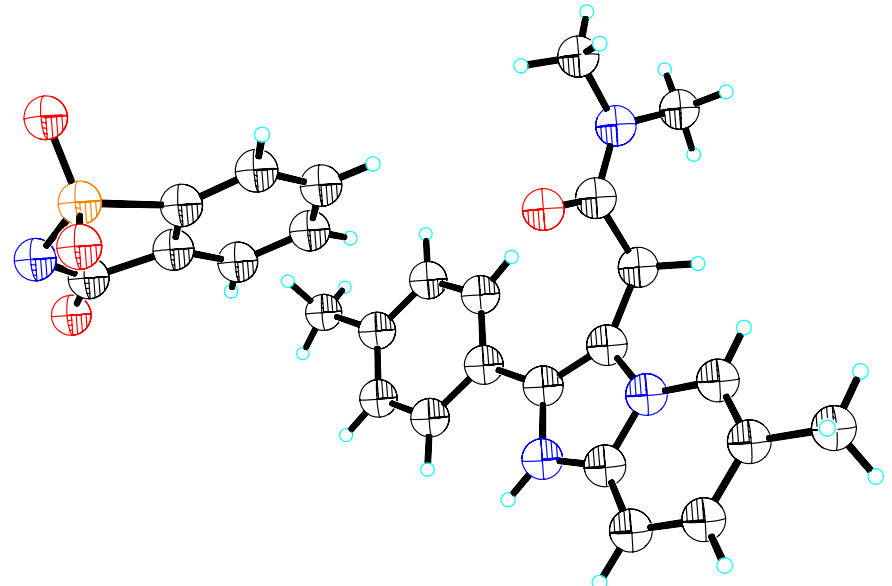

(ii)

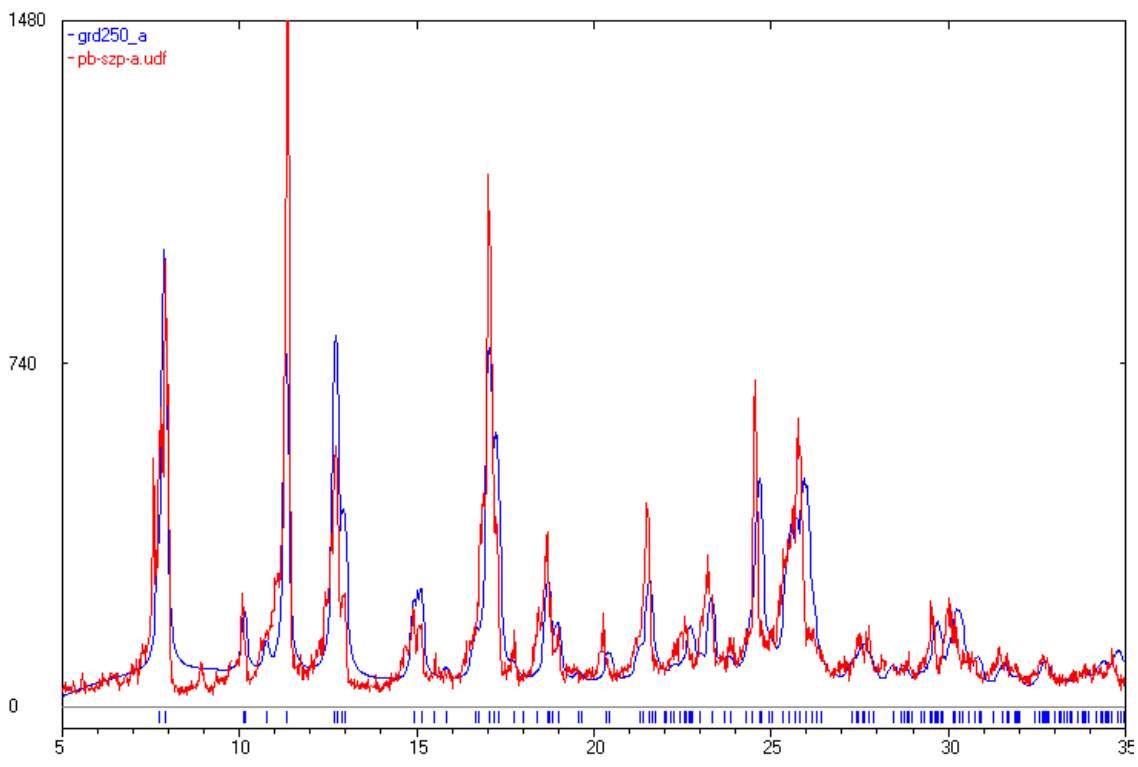


(iii)

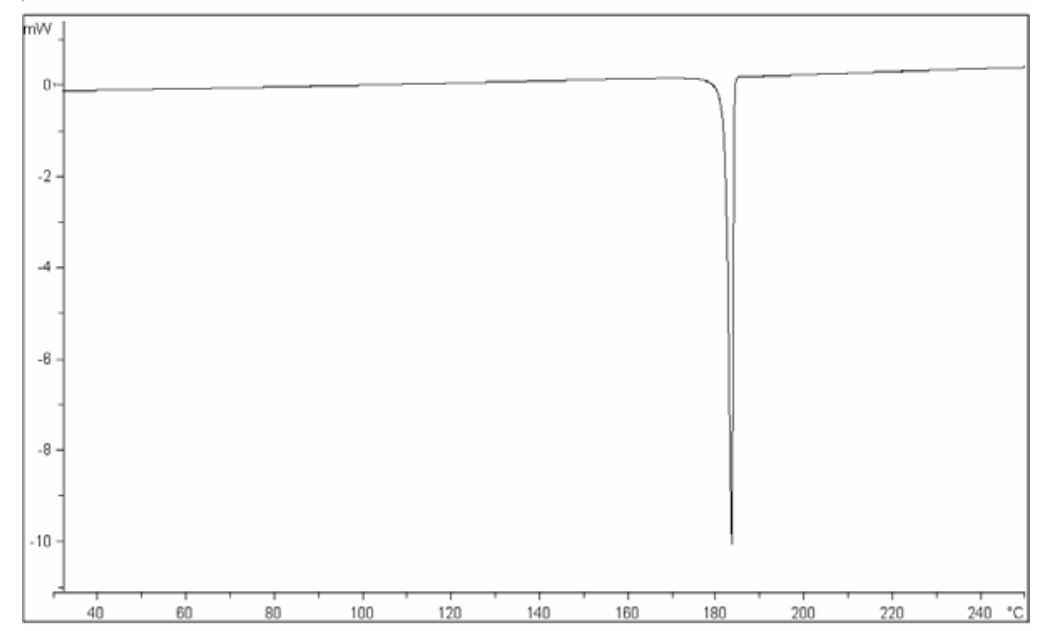

(iv)

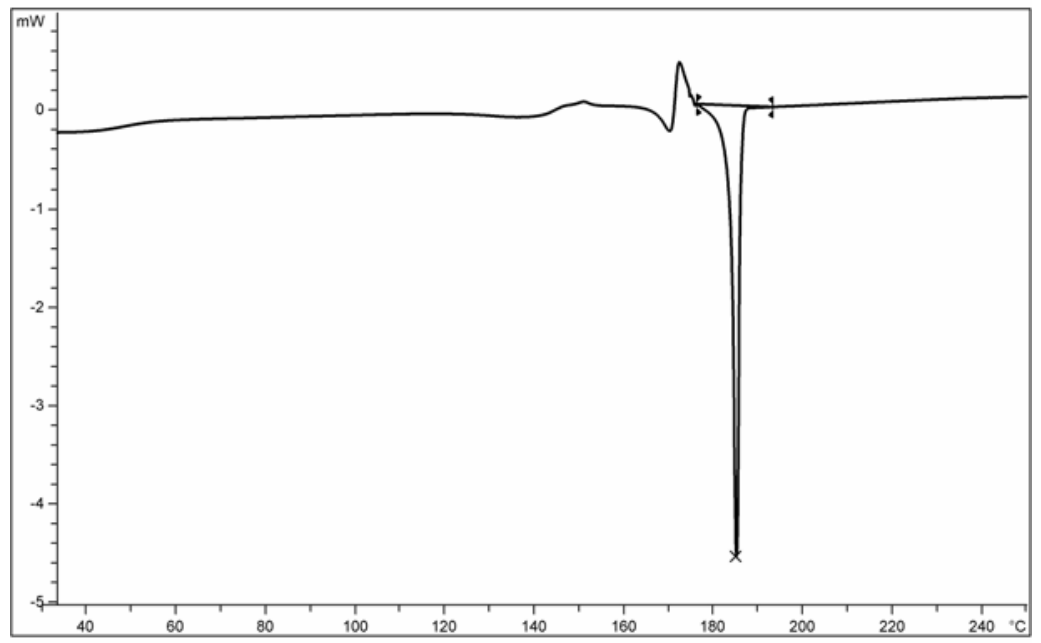

(v)

Saccharin $\left(\mathrm{cm}^{-1}\right)$ : 3396, 3094, 2972, 2690, 2010, 1975, 1865, 1720, 1593, 1460, 1336, $1255,1178,1138,1118,1008,972,898,794,758,702$.

Zolpidem (cm $\left.{ }^{-1}\right)$ : 2937, 2912, 1635, 1537, 1504, 1394, 1342, 1263, 1186, 1138, 823, 792.

Zolpidem saccharinate (before solubility) $\left(\mathrm{cm}^{-1}\right)$ : 3051, 2926, 2598, 1655, 1604, 1560, 1398, 1361, 1284, 1149, 1051, 912, 810, 752.

Zolpidem saccharinate (after solubility) $\left(\mathrm{cm}^{-1}\right)$ : 3470, 3057, 2924, 2598, 1655, 1606, 1560, 1398, 1348, 1284, 1151, 1051, 914, 812, 752. 
Sertraline saccharinate

(i)

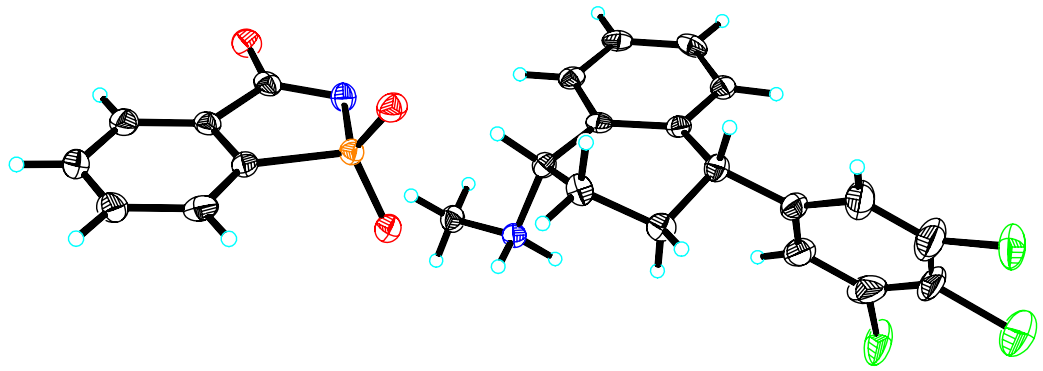

(ii)

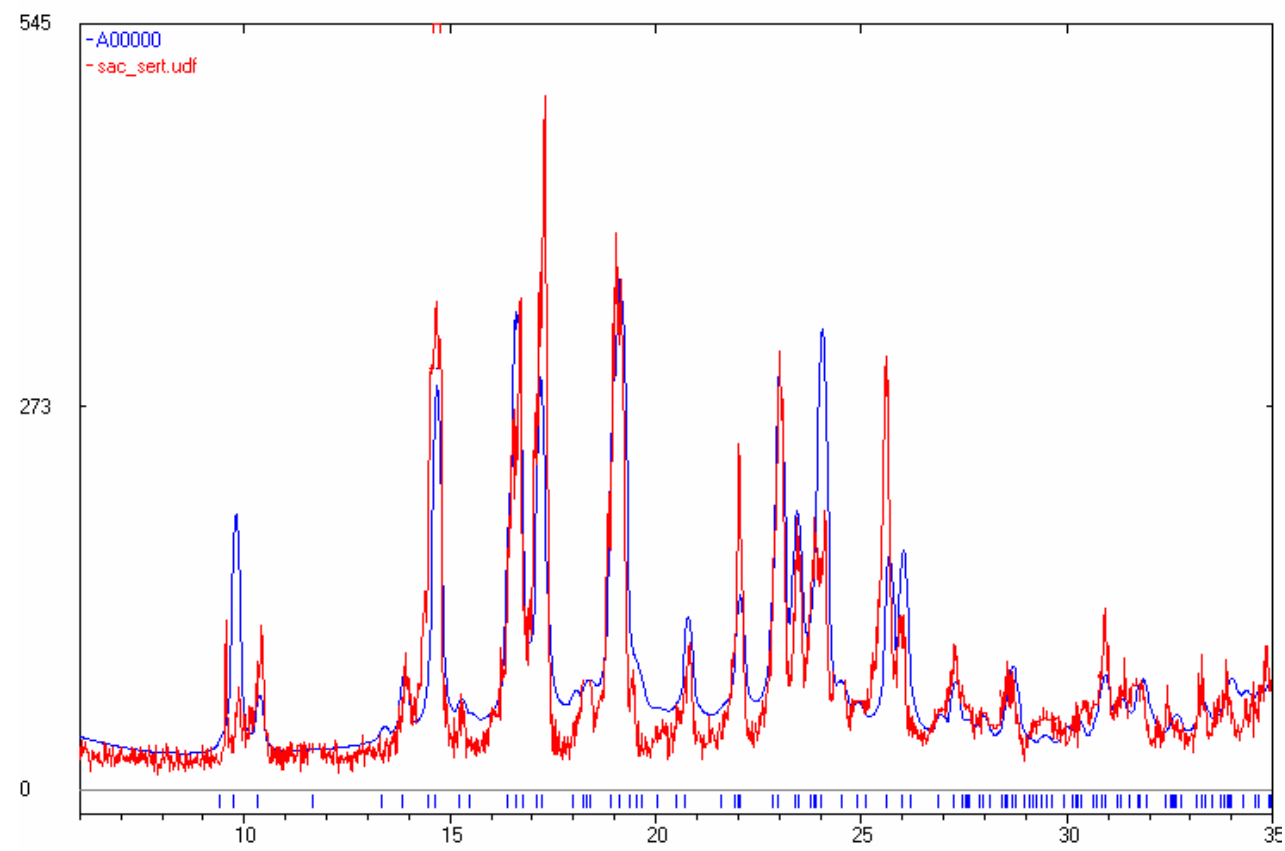


(iii)

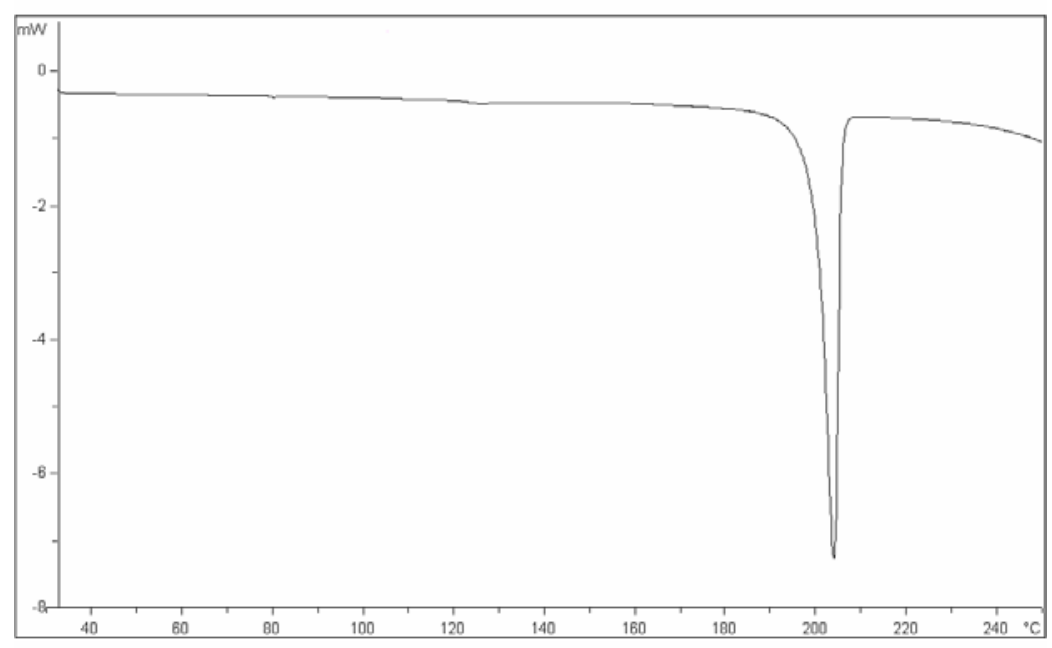

(iv)

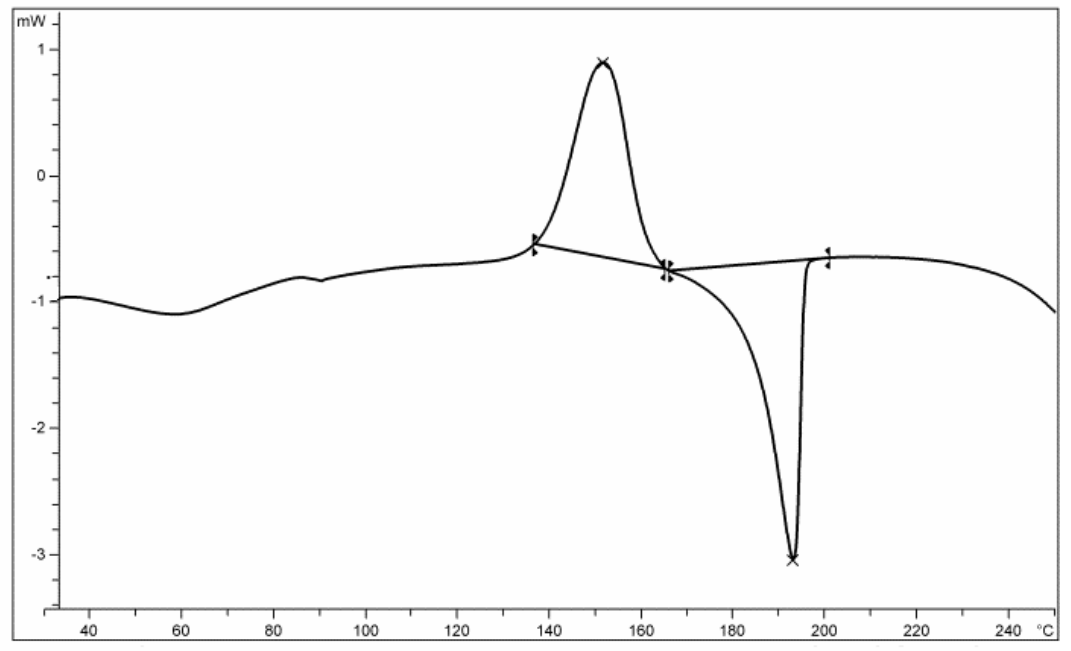

(v)

Saccharin $\left(\mathrm{cm}^{-1}\right)$ : 3396, 3094, 2972, 2690, 2010, 1975, 1865, 1720, 1593, 1460, 1336, 1255, 1178, 1138, 1118, 1008, 972, 898, 794, 758, 702.

Sertaline $\left(\mathrm{cm}^{-1}\right)$ : 2930, 2845, 2781, 1556, 1496, 1471, 1396, 1128, 873, 827.

Sertaline saccharinate (before solubility) $\left(\mathrm{cm}^{-1}\right)$ : 3173, 2955, 2874, 2683, 2459, 1892, 1826, 1616,1575, 1483, 1454, 1402, 1331, 1255, 1153, 1049, 945, 883, 785.

Sertaline saccharinate (after solubility) $\left(\mathrm{cm}^{-1}\right)$ : 3173, 2954, 2872, 2683, 2459, 1892, 1826,1616,1573, 1483, 1454, 1402, 1331, 1255, 1153, 1049, 945, 883, 785. 
Amlodipine saccharinate

(i)

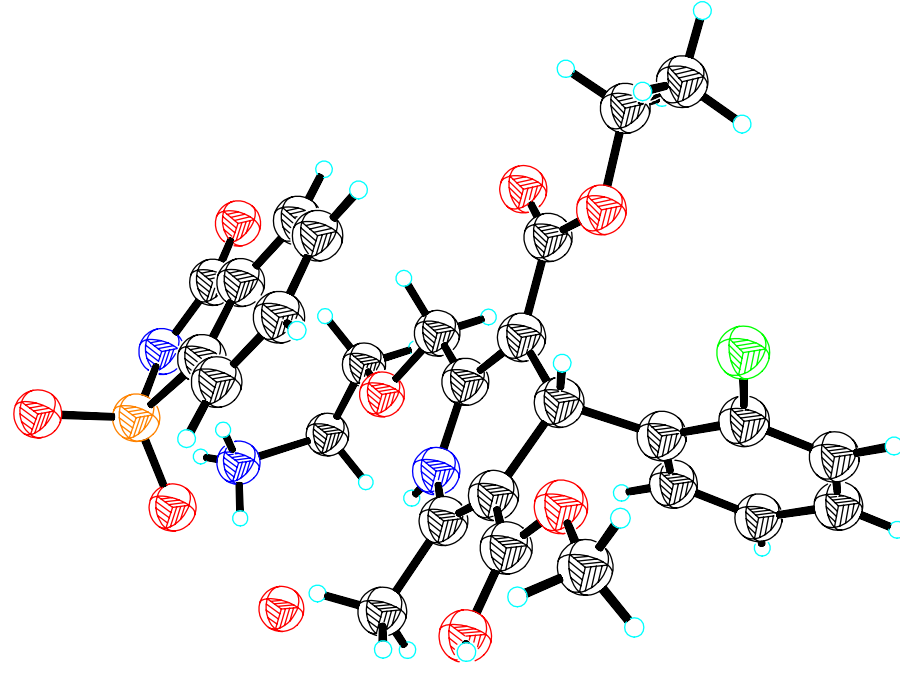


Quinine saccharinate:

(i)

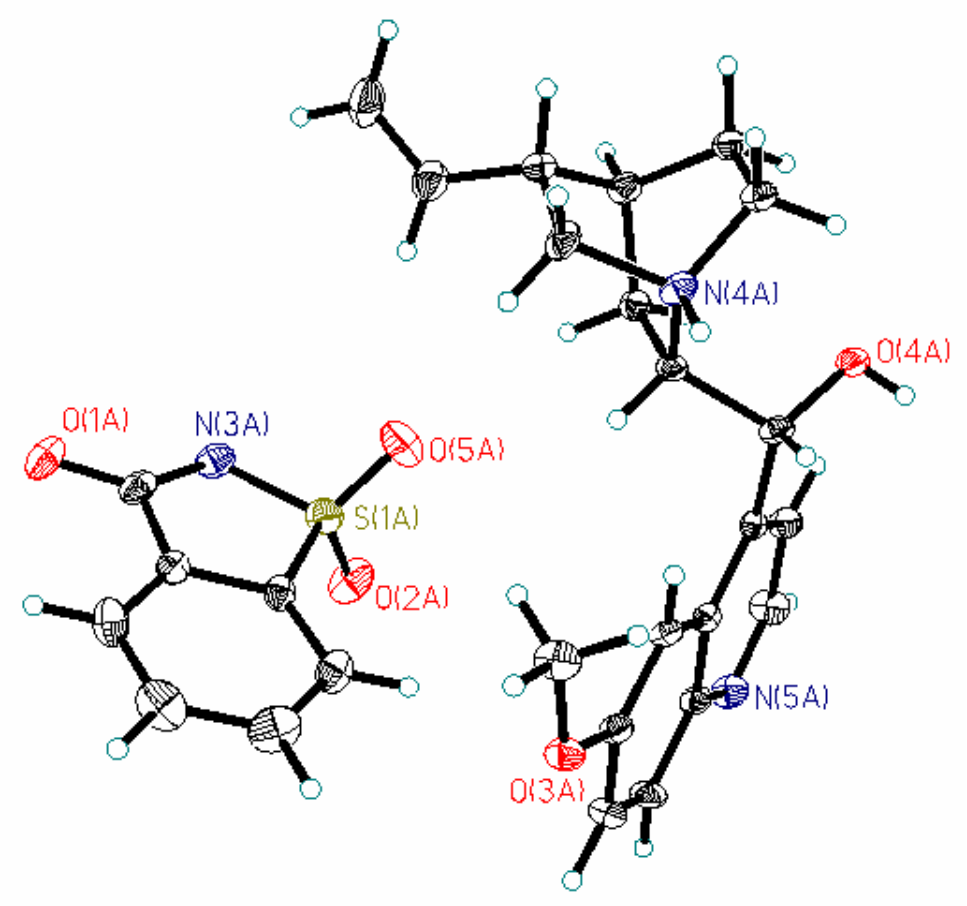

(ii)

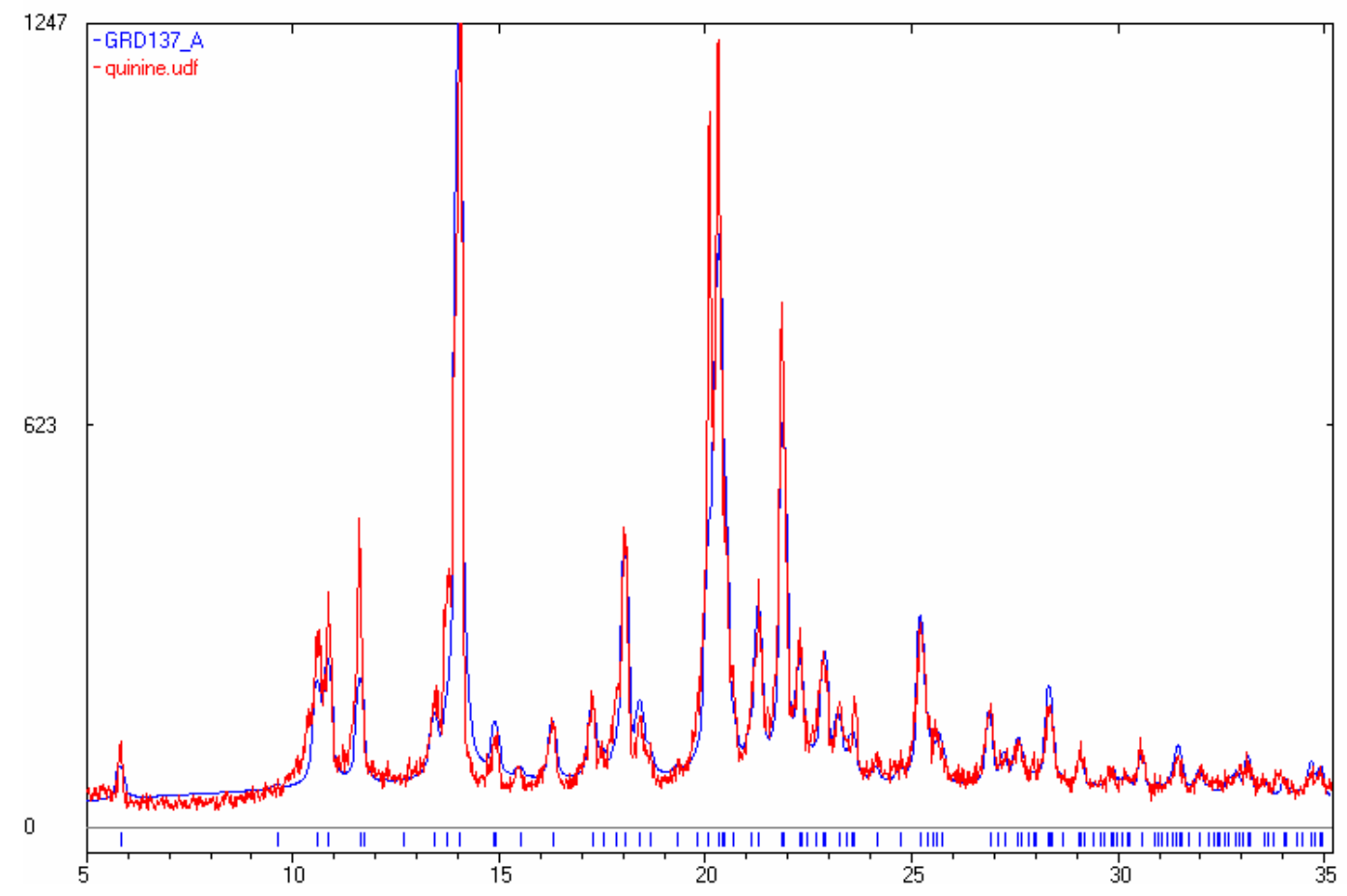


(iii)

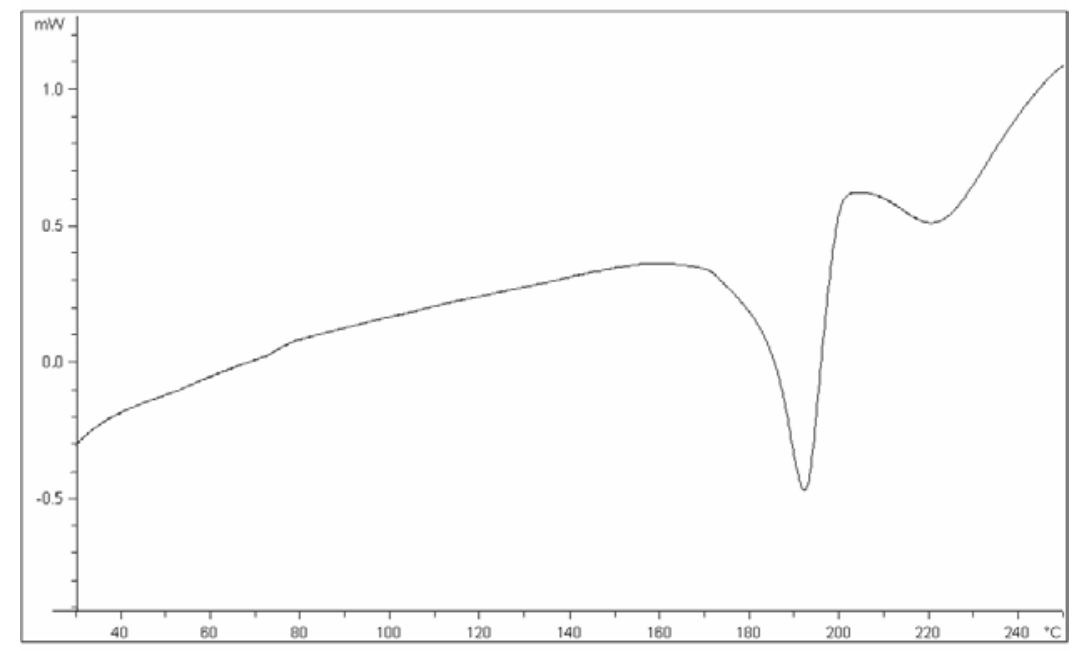

(iv)

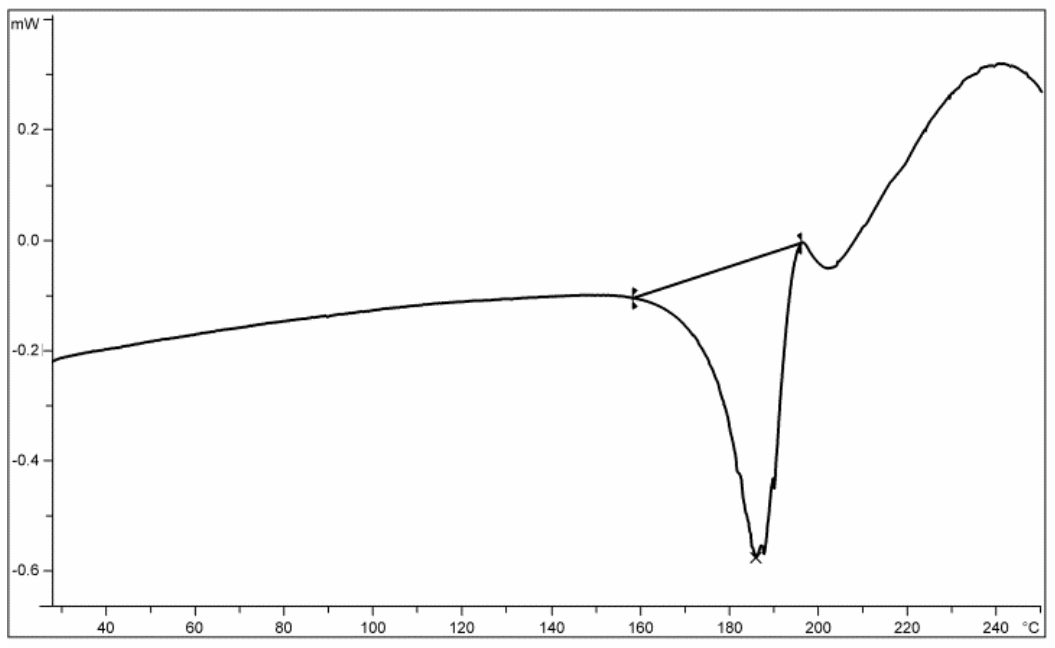

(v)

Saccharin $\left(\mathrm{cm}^{-1}\right)$ : 3396, 3094, 2972, 2690, 2010, 1975, 1865, 1720, 1593, 1460, 1336, 1255, 1178, 1138, 1118, 1008, 972, 898, 794, 758, 702.

Quinine $\left(\mathrm{cm}^{-1}\right)$ : 3148, 2928, 1927, 1622, 1589, 1508, 1469, 1431, 1240, 1136, 1103, 1030, 904, 883, 854, 821.

Quinine saccharinate (before solubility) $\left(\mathrm{cm}^{-1}\right)$ : 3074, 2833, 1651, 1589, 1512, 1456, 1431, 1325, 1280, 1246, 1149, 1030, 945, 860, 829, 754.

Quinine saccharinate (after solubility) $\left(\mathrm{cm}^{-1}\right)$ : 3072, 2838, 1657, 1589, 1512, 1446, 1431, 1320, 1280, 1141, 1030, 864, 829, 754. 
Mirtazapine saccharinate:

(i)

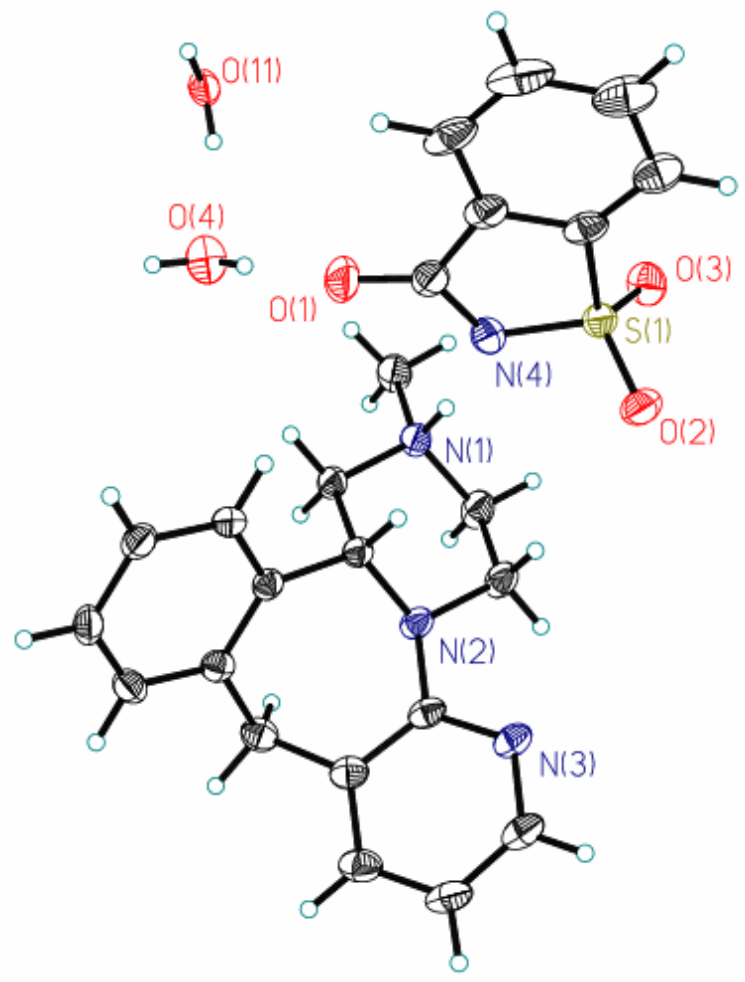

(ii)

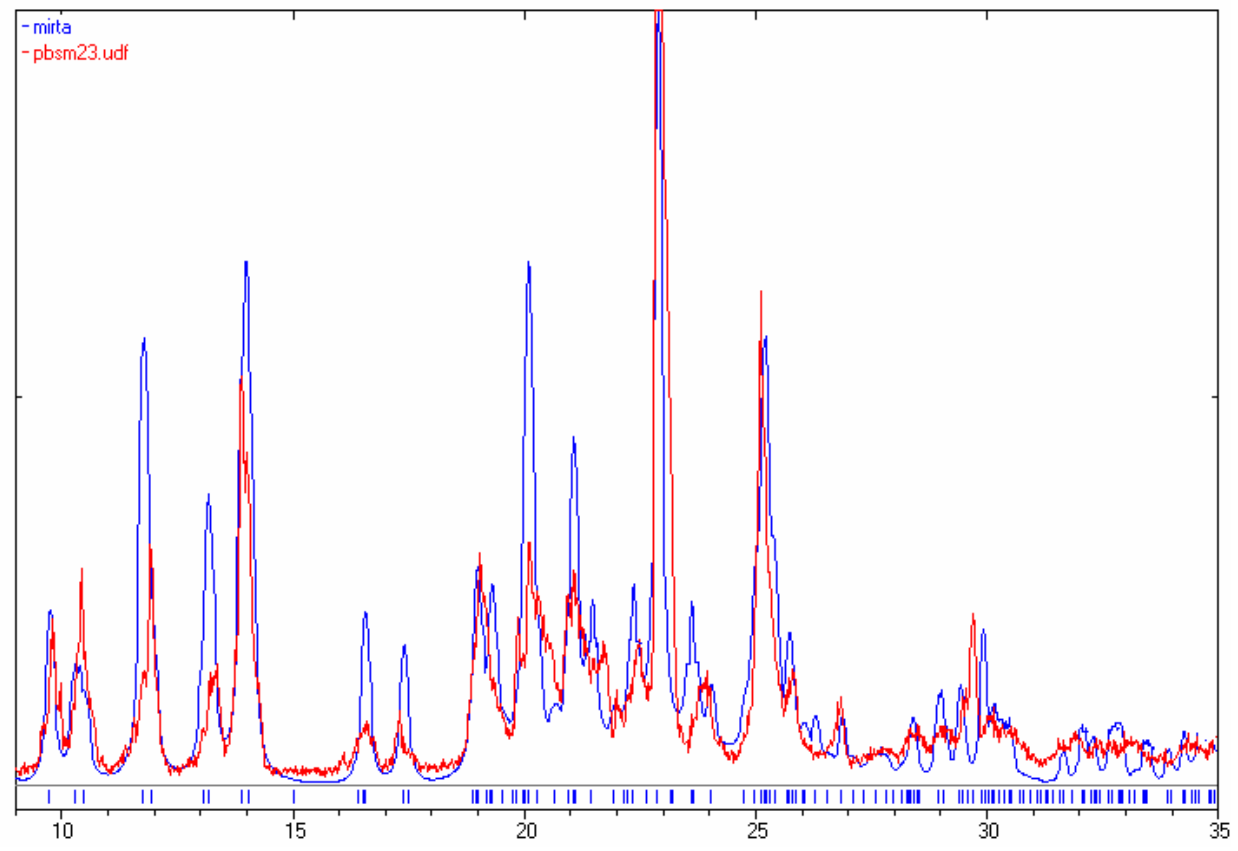


(iii)

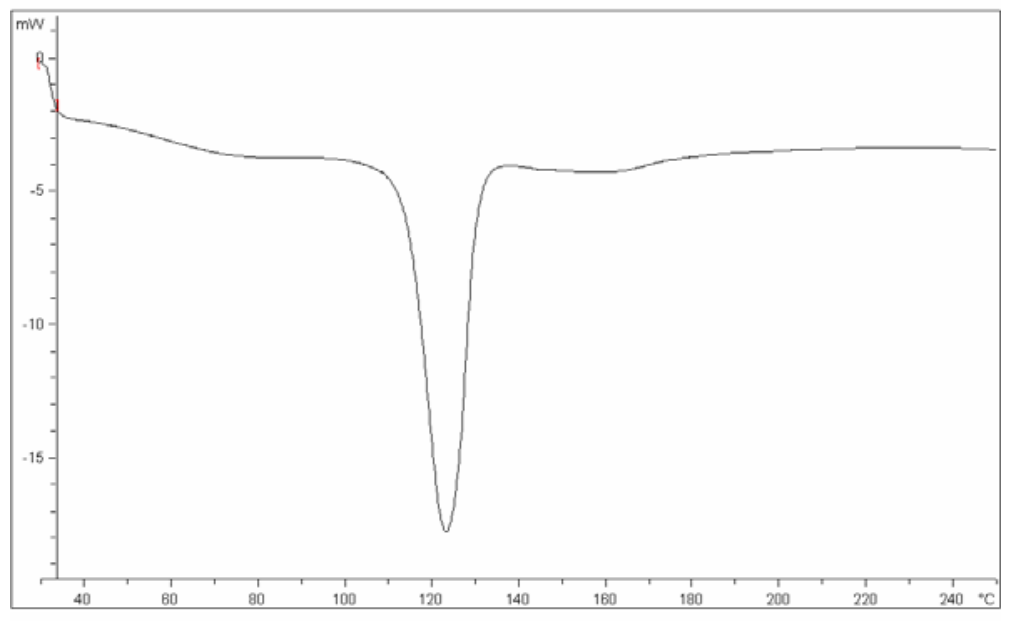

(iv)

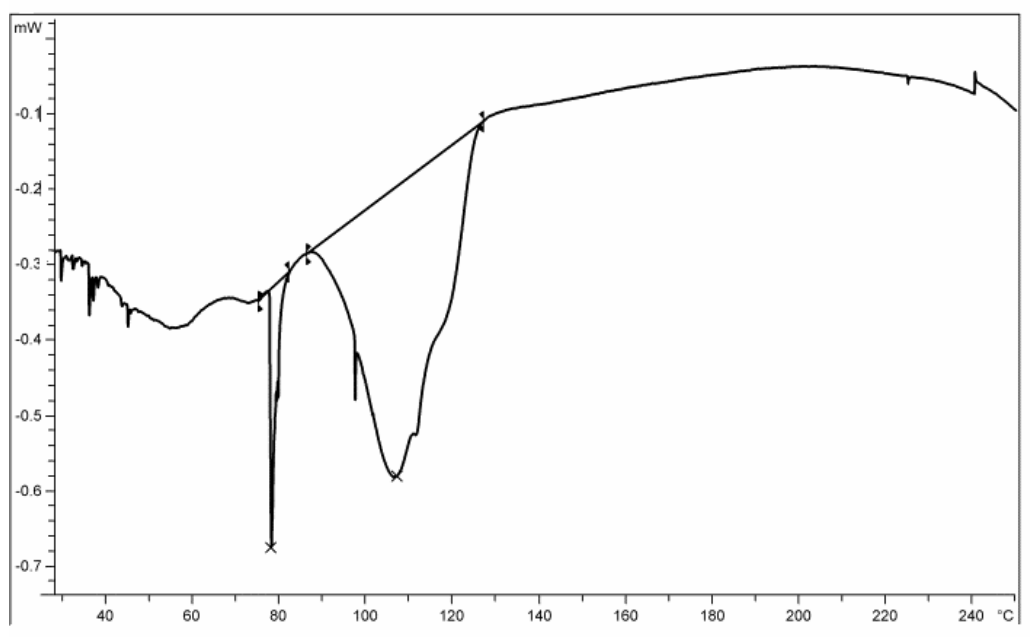

(v)

Saccharin $\left(\mathrm{cm}^{-1}\right)$ : 3396, 3094, 2972, 2690, 2010, 1975, 1865, 1720, 1593, 1460, 1336, 1255, 1178, 1138, 1118, 1008, 972, 898, 794, 758, 702.

Mirtazapine ( $\left.\mathrm{cm}^{-1}\right)$ : 3425, 2966, 2932, 2804, 1585, 1564, 1496, 1444, 1388, 1359, 1336, 1288, 1253, 1222, 1190, 1138, 1122, 1072, 1020, 995, 960.

Mirtazapine saccharinate (before solubility) $\left(\mathrm{cm}^{-1}\right)$ : 3528, 3057, 2916, 2856, 2391, 1649, 1587, 1496, 1440, 1381, 1332, 1282, 1251, 1151, 1111, 952.

Mirtazapine saccharinate (after solubility) $\left(\mathrm{cm}^{-1}\right)$ : 3535, 3057, 2916, 2856, 2359 (b), 1649, 1587, 1494, 1440, 1381, 1332, 1282, 1251, 1151, 1111, 1051, 952. 


\section{Piroxicam saccharinate:}

(i)

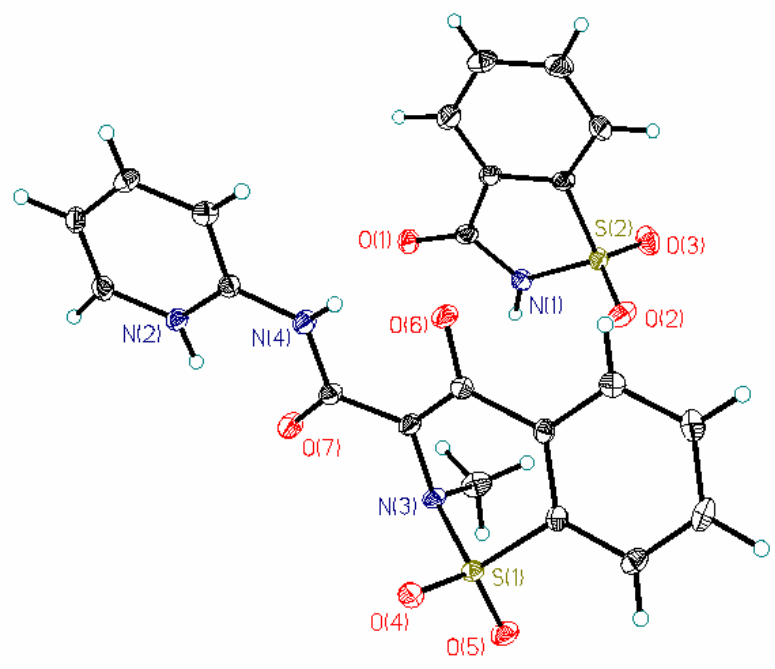

(ii)

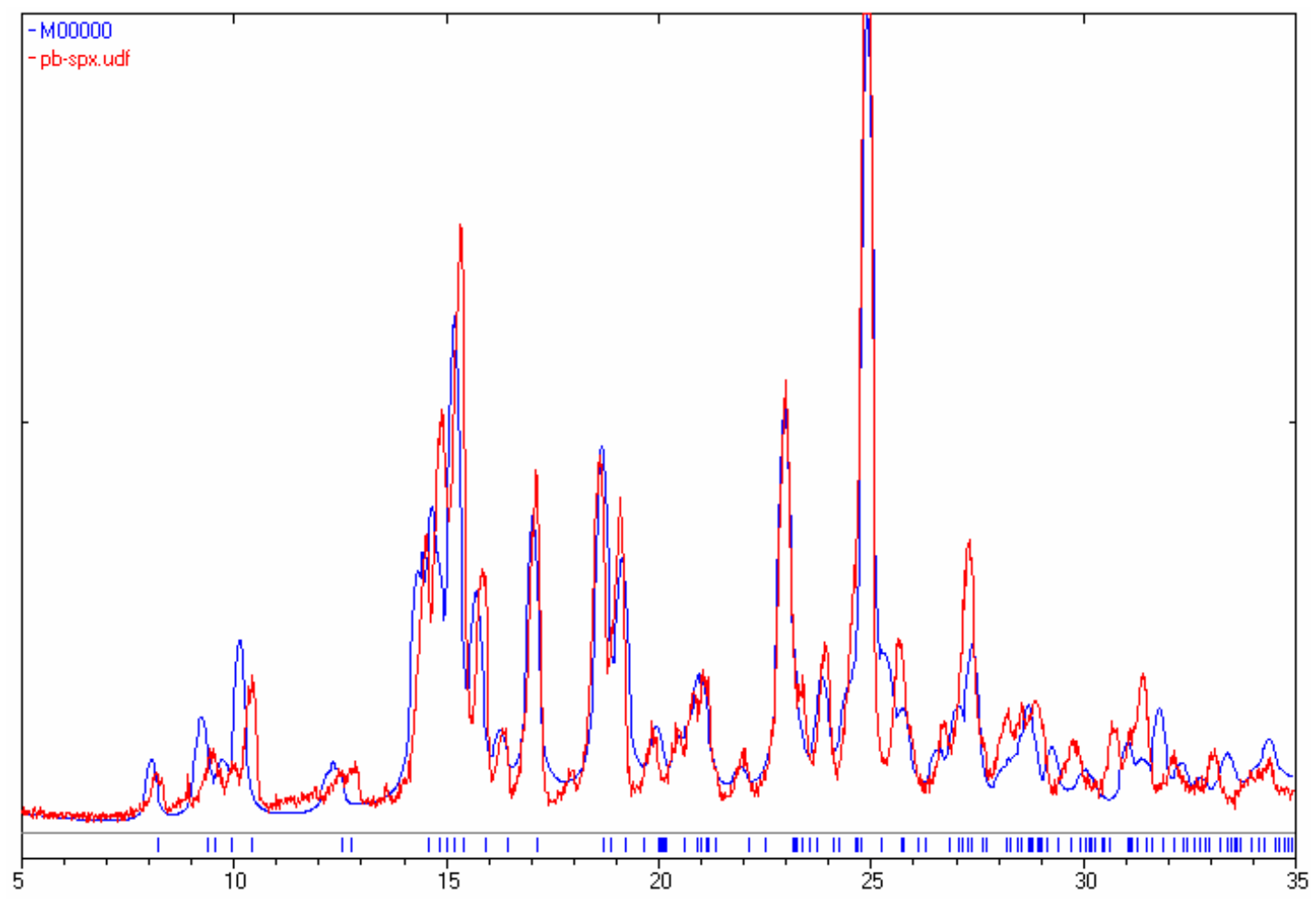




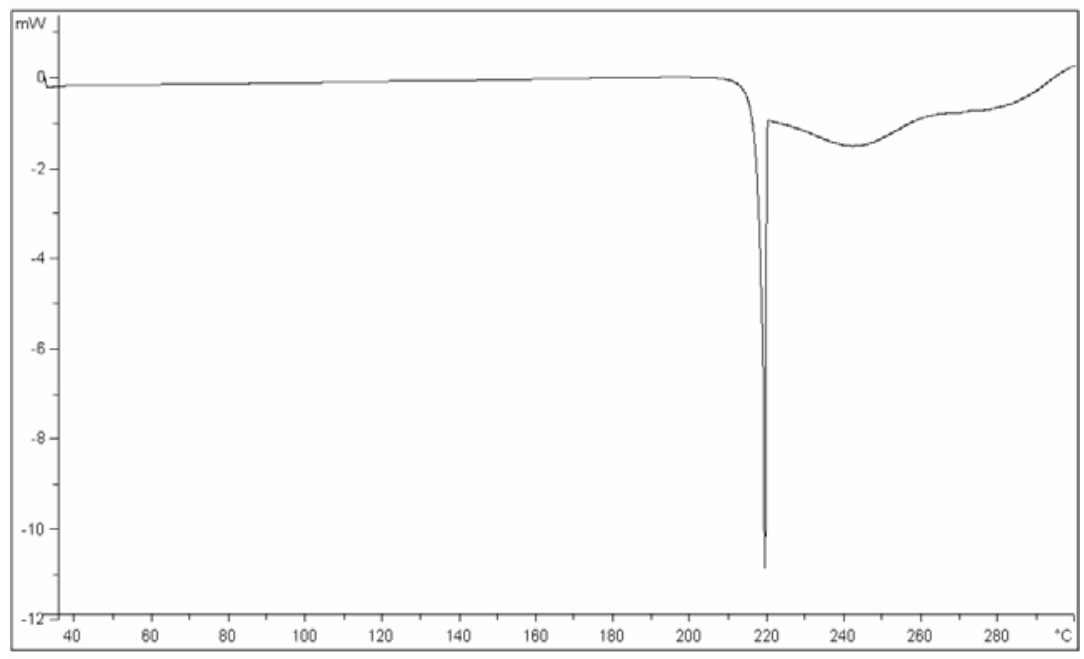

(iv)

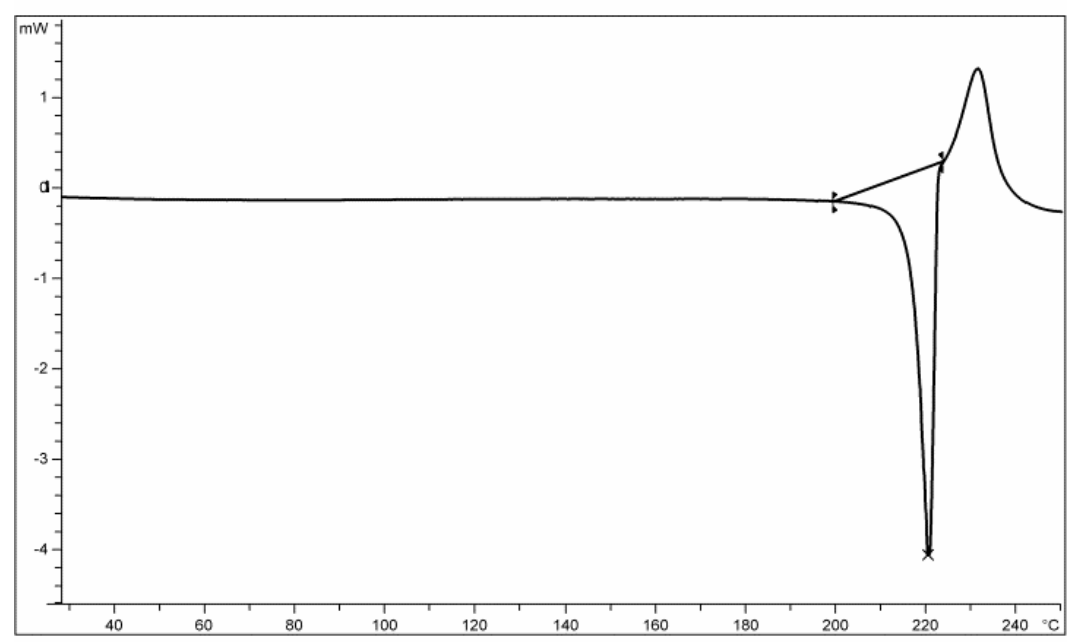

(v)

Saccharin $\left(\mathrm{cm}^{-1}\right)$ : 3396, 3094, 2972, 2690, 2010, 1975, 1865, 1720, 1593, 1460, 1336, 1255, 1178, 1138, 1118, 1008, 972, 898, 794, 758, 702.

Piroxicam ( $\left.\mathrm{cm}^{-1}\right)$ : 3337, 1630, 1577, 1529, 1435, 1350, 1300, 1215, 1180, 1149, 1039, 937, 875, 829, 773, 731.

Piroxicam saccharin co-crystal (before solubility) (cm ${ }^{-1}$ ): 3078, 2876, 2598, 1736, 1641, 1608, 1554, 1514, 1433, 1406, 1332, 1240, 1159, 1116, 1095, 999, 906, 875, 846, 769.

Piroxicam saccharin co-crystal (after solubility) $\left(\mathrm{cm}^{-1}\right)$ : 3078, 2878, 2586, 1736, 1641, 1606, 1554, 1516, 1433, 1406, 1331, 1240, 1159, 1116, 906, 873, 814, 767. 
Mirtazapine saccharinate, hot stage microscope photographs

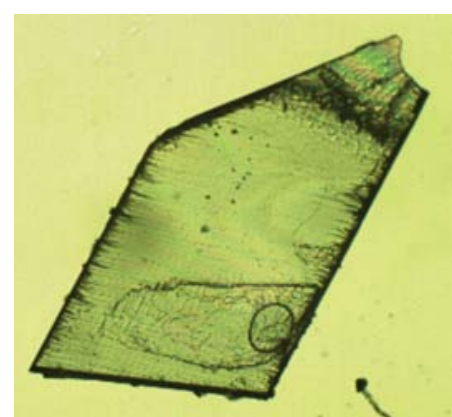

$29^{\circ} \mathrm{C}$

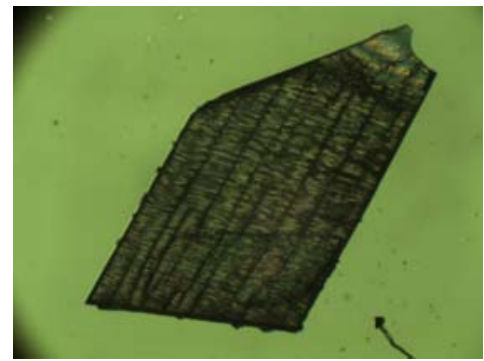

$96^{\circ} \mathrm{C}$

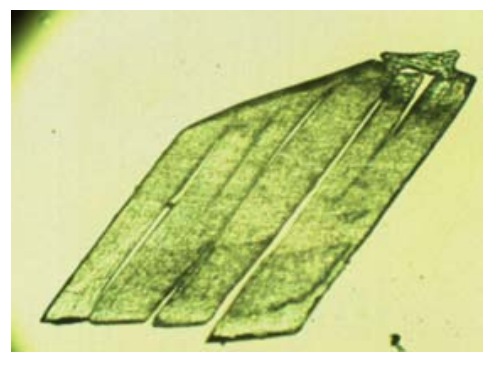

$119^{\circ} \mathrm{C}$

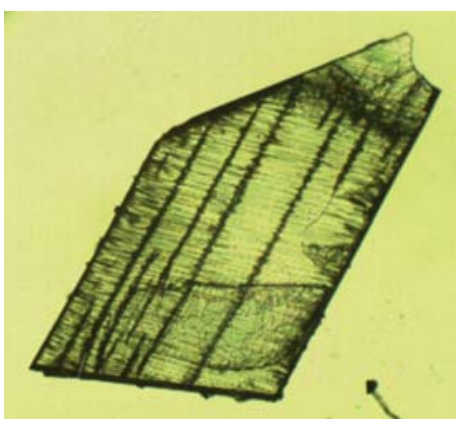

$34^{\circ} \mathrm{C}$

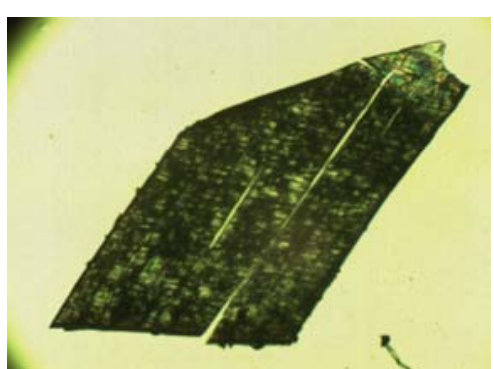

$112^{\circ} \mathrm{C}$

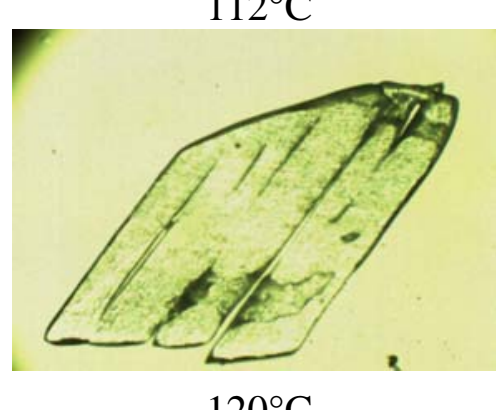

$120^{\circ} \mathrm{C}$

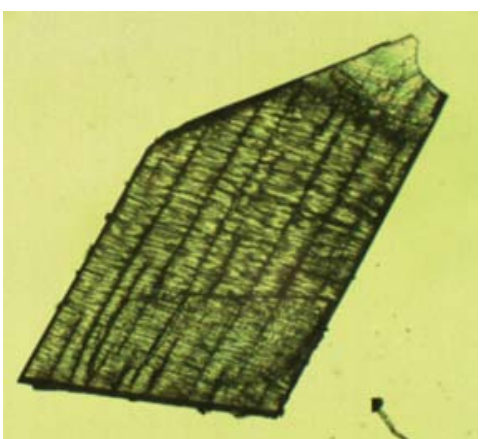

$50^{\circ} \mathrm{C}$

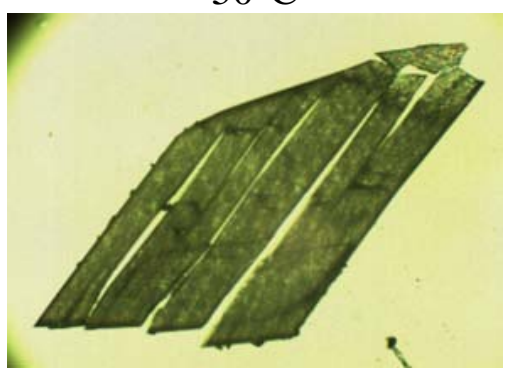

$114^{\circ} \mathrm{C}$

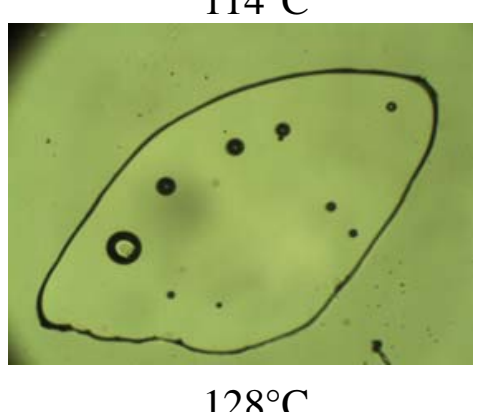

